\title{
THIRD-ORDER PERTURBATION THEORY WITH NONLINEAR PRESSURE
}

\author{
Masatoshi Shoji and EiIChiro Komatsu \\ Texas Cosmology Center, University of Texas at Austin, 1 University Station, C1400 Austin, TX 78712, USA; mshoji @astro.as.utexas.edu \\ Received 2009 March 15; accepted 2009 May 21; published 2009 July 6
}

\begin{abstract}
We calculate the nonlinear matter power spectrum using the third-order perturbation theory without ignoring the pressure gradient term. We consider a semirealistic system consisting of two matter components with and without pressure, and both are expanded into the third order in perturbations in a self-consistent manner, for the first time. While the pressured component may be identified with baryons or neutrinos, in this paper we mainly explore the physics of the nonlinear pressure effect using a toy model in which the Jeans length does not depend on time, i.e., the sound speed decreases as $a^{-1 / 2}$, where $a$ is the scale factor. The linear analysis shows that the power spectrum below the so-called filtering scale is suppressed relative to the power spectrum of the cold dark matter. Our nonlinear calculation shows that the actual filtering scale for a given sound speed is smaller than the linear filtering scale by a factor depending on the redshift and the Jeans length. A $\sim 40 \%$ change is common, and our results suggest that, when applied to baryons, the temperature of the intergalactic medium inferred from the filtering scale observed in the flux power spectrum of Ly $\alpha$ forests would be underestimated by a factor of 2 , if one used the linear filtering scale to interpret the data. The filtering mass, which is proportional to the filtering scale cubed, can also be significantly smaller than the linear theory prediction especially at low redshift, where the actual filtering mass can be smaller than the linear prediction by a factor of 3. Finally, when applied to neutrinos, we find that neutrino perturbations deviate significantly from linear perturbations even below the free-streaming scales, and thus neutrinos cannot be treated as linear perturbations.
\end{abstract}

Key words: cosmology: theory - large-scale structure of universe

Online-only material: color figure

\section{INTRODUCTION}

Pressure plays an important role for the structure formation in the universe. Pressure determines the Jeans scale, $\lambda_{J}$, below which the growth of structure slows down, and eventually stops and oscillates: while fluctuations in the cold dark matter (CDM) and the pressured component evolve in the same way above the Jeans scale, their evolutions are significantly different below the Jeans scale.

The dominant source of gravity is CDM, which is cold and its velocity dispersion is negligible before the collapse of halos. However, the subdominant matter componentsbaryons and neutrinos-have significant velocity dispersions, which should be included in the calculation when precision is required. While the accurate calculations have been done for the linear perturbations, the effects of the pressure on the nonlinear evolution of matter fluctuations on cosmological scales $(\sim 10-100 \mathrm{Mpc})$ have not been studied very much in the literature.

We address this issue by calculating the nonlinear matter power spectrum using the third-order perturbation theory (3PT; see Bernardeau et al. 2002, for a review), with the pressure gradient term in the Euler equation explicitly included. This enables us to study the effects of the pressure on the nonlinear evolution of matter fluctuations in a self-consistent manner.

The rest of this paper is organized as follows. In Section 2, we find the linear, second-order, and third-order solutions of the coupled continuity, Euler, and Poisson equations for two matter components with and without the pressure gradient. In Section 3, we calculate the nonlinear matter power spectrum from the solutions obtained in Section 2. In Section 4, we compare our full 3PT calculation with the approximation used by Saito et al. (2008) for the effects of massive neutrinos on the matter power spectrum. Finally, in Section 5, we discuss the implications of our results for a few practical astrophysical and cosmological applications. In the appendices we give the detailed derivations of the 3PT results used in the main body of the paper.

\section{THIRD-ORDER PERTURBATION THEORY WITH PRESSURE}

\subsection{Basic Equations}

The main goal of this paper is to find the perturbative solutions for the CDM density contrast, $\delta_{c}$, for which the pressure gradient is ignored, and the density contrast of another matter component, $\delta_{b}$, for which the pressure gradient is retained. This component may be identified with baryons (hence the subscript " $b$ ") or neutrinos, depending on the sound speed one uses in the Euler equation. ${ }^{1}$

The equations that we are going to solve include two continuity equations

$$
\begin{aligned}
& \dot{\delta}_{c}(\mathbf{x}, \tau)+\nabla \cdot\left[\left(1+\delta_{c}(\mathbf{x}, \tau)\right) \mathbf{v}_{c}(\mathbf{x}, \tau)\right]=0, \\
& \dot{\delta}_{b}(\mathbf{x}, \tau)+\nabla \cdot\left[\left(1+\delta_{b}(\mathbf{x}, \tau)\right) \mathbf{v}_{b}(\mathbf{x}, \tau)\right]=0,
\end{aligned}
$$

two Euler equations

$$
\dot{\mathbf{v}}_{c}(\mathbf{x}, \tau)+\left[\mathbf{v}_{c}(\mathbf{x}, \tau) \cdot \nabla\right] \mathbf{v}_{c}(\mathbf{x}, \tau)=-\frac{\dot{a}}{a} \mathbf{v}_{c}(\mathbf{x}, \tau)-\nabla \phi(\mathbf{x}, \tau),
$$

\footnotetext{
1 While we use " $b$ " to denote the pressured matter component throughout this paper, we do not always mean baryons, but we always refer to a general matter component with pressure.
} 


$$
\begin{aligned}
& \dot{\mathbf{v}}_{b}(\mathbf{x}, \tau)+\left[\mathbf{v}_{b}(\mathbf{x}, \tau) \cdot \nabla\right] \mathbf{v}_{b}(\mathbf{x}, \tau) \\
& =-\frac{\dot{a}}{a} \mathbf{v}_{b}(\mathbf{x}, \tau)-\nabla \phi(\mathbf{x}, \tau)-\frac{c_{s}^{2}(\mathbf{x}, \tau) \nabla \delta_{b}(\mathbf{x}, \tau)}{1+\delta_{b}(\mathbf{x}, \tau)},
\end{aligned}
$$

and one Poisson equation

$$
\nabla^{2} \phi(\mathbf{x}, \tau)=4 \pi G a^{2}\left[\bar{\rho}_{c}(\tau) \delta_{c}(\mathbf{x}, \tau)+\bar{\rho}_{b}(\tau) \delta_{b}(\mathbf{x}, \tau)\right],
$$

where $\delta_{i} \equiv\left(\rho_{i}-\bar{\rho}_{i}\right) / \bar{\rho}_{i}$ is the density contrast of a matter component $i=(c, b), \bar{\rho}$ is the background matter density, $a$ is the scale factor, $\mathbf{v}_{i}$ is the peculiar velocity field of a matter component $i, \phi$ is the gravitational potential, and $c_{s}$ is the sound speed of the matter component with pressure. Here, the dots denote the partial derivatives with respect to the conformal time, $\tau$, i.e., $\dot{\delta}=\partial \delta / \partial \tau$, and $\nabla$ denotes the partial derivatives with respect to the comoving coordinates.

We rewrite the Poisson equation as

$$
\nabla^{2} \phi(\mathbf{x}, \tau)=\frac{6}{\tau^{2}} \delta(\mathbf{x}, \tau)
$$

where we have assumed an Einstein-de Sitter (EdS) universe (we shall generalize the results to other cosmological models later), for which the energy density of the universe is dominated entirely by the matter density, and $a \propto \tau^{2}$. The background Friedmann equation is given by

$$
\frac{8 \pi G}{3}\left[\bar{\rho}_{c}(\tau)+\bar{\rho}_{b}(\tau)\right] a^{2}=\frac{4}{\tau^{2}} .
$$

We have also defined the total matter fluctuation, $\delta$, which is given by

$$
\begin{aligned}
\delta(\mathbf{x}, \tau) & \equiv \frac{\bar{\rho}_{c}(\tau) \delta_{c}(\mathbf{x}, \tau)+\bar{\rho}_{b}(\tau) \delta_{b}(\mathbf{x}, \tau)}{\bar{\rho}_{c}(\tau)+\bar{\rho}_{b}(\tau)} \\
& =f_{c} \delta_{c}(\mathbf{x}, \tau)+f_{b} \delta_{b}(\mathbf{x}, \tau)
\end{aligned}
$$

where $f_{c} \equiv \bar{\rho}_{c} /\left(\bar{\rho}_{c}+\bar{\rho}_{b}\right)=\Omega_{c} / \Omega_{m}$, and $f_{b} \equiv \bar{\rho}_{b} /\left(\bar{\rho}_{c}+\bar{\rho}_{b}\right)=$ $\Omega_{b} / \Omega_{m}$. For an EdS universe, $\Omega_{m}=1$.

Taking the divergence of the Euler equations, we obtain the equations for the velocity divergence fields, $\theta_{i} \equiv \nabla \cdot \mathbf{v}_{i}$. Moving nonlinear terms to the right-hand side of the equations and using the Poisson equation, we obtain

$$
\begin{gathered}
\dot{\delta}_{c}(\mathbf{x}, \tau)+\theta_{c}(\mathbf{x}, \tau)=-\nabla \cdot\left[\delta_{c}(\mathbf{x}, \tau) \mathbf{v}_{c}(\mathbf{x}, \tau)\right], \\
\dot{\delta}_{b}(\mathbf{x}, \tau)+\theta_{b}(\mathbf{x}, \tau)=-\nabla \cdot\left[\delta_{b}(\mathbf{x}, \tau) \mathbf{v}_{b}(\mathbf{x}, \tau)\right], \\
\dot{\theta}_{c}(\mathbf{x}, \tau)+\frac{2}{\tau} \theta_{c}(\mathbf{x}, \tau)+\frac{6}{\tau^{2}} \delta(\mathbf{x}, \tau)=-\nabla \cdot\left\{\left[\mathbf{v}_{c}(\mathbf{x}, \tau) \cdot \nabla\right] \mathbf{v}_{c}(\mathbf{x}, \tau)\right\}, \\
\dot{\theta}_{b}(\mathbf{x}, \tau)+\frac{2}{\tau} \theta_{b}(\mathbf{x}, \tau)+\frac{6}{\tau^{2}} \delta(\mathbf{x}, \tau) \\
=-\nabla \cdot\left\{\left[\mathbf{v}_{b}(\mathbf{x}, \tau) \cdot \nabla\right] \mathbf{v}_{b}(\mathbf{x}, \tau)\right\}-\nabla \cdot\left[\frac{c_{s}^{2}(\mathbf{x}, \tau) \nabla \delta_{b}(\mathbf{x}, \tau)}{1+\delta_{b}(\mathbf{x}, \tau)}\right] .
\end{gathered}
$$

Note that the second term on the right-hand side of Equation (12) still contains the linear order term. All the other terms on the right-hand side of the above equations are nonlinear.
We shall simplify the pressure term, the second term on the right-hand side of Equation (12), as follows. First, we shall assume that the sound speed is homogeneous, i.e., $\nabla c_{s}^{2}=0$. See Naoz \& Barkana (2005) for the analysis of linear perturbations with $\nabla c_{s}^{2} \neq 0$. Second, we expand the pressure term to the third order in perturbations:

$$
\frac{\nabla \delta \rho_{b}}{\rho_{b}}=\frac{\nabla \delta_{b}}{1+\delta_{b}} \simeq \nabla \delta_{b}-\delta_{b} \nabla \delta_{b}+\delta_{b}^{2} \nabla \delta_{b}+\mathcal{O}\left(\delta_{b}^{4}\right) .
$$

Going to Fourier space, we obtain

$$
\begin{aligned}
\dot{\tilde{\delta}}_{c}(\mathbf{k}, \tau)+\tilde{\theta}_{c}(\mathbf{k}, \tau)= & -\frac{1}{(2 \pi)^{3}} \iint d \mathbf{q}_{1} d \mathbf{q}_{2} \delta_{D}\left(\mathbf{q}_{1}+\mathbf{q}_{2}-\mathbf{k}\right) \\
& \times \frac{\mathbf{k} \cdot \mathbf{q}_{1}}{q_{1}^{2}} \tilde{\theta}_{c}\left(\mathbf{q}_{1}, \tau\right) \tilde{\delta}_{c}\left(\mathbf{q}_{2}, \tau\right),
\end{aligned}
$$

$$
\begin{aligned}
\dot{\tilde{\delta}}_{b}(\mathbf{k}, \tau)+\tilde{\theta}_{b}(\mathbf{k}, \tau)= & -\frac{1}{(2 \pi)^{3}} \iint d \mathbf{q}_{1} d \mathbf{q}_{2} \delta_{D}\left(\mathbf{q}_{1}+\mathbf{q}_{2}-\mathbf{k}\right) \\
& \times \frac{\mathbf{k} \cdot \mathbf{q}_{1}}{q_{1}^{2}} \tilde{\theta}_{b}\left(\mathbf{q}_{1}, \tau\right) \tilde{\delta}_{b}\left(\mathbf{q}_{2}, \tau\right),
\end{aligned}
$$

$$
\begin{aligned}
\dot{\tilde{\theta}}_{c}(\mathbf{k}, \tau)+\frac{2}{\tau} \tilde{\theta}_{c}(\mathbf{k}, \tau)+\frac{6}{\tau^{2}} \tilde{\delta}(\mathbf{k}, \tau) \\
=-\frac{1}{(2 \pi)^{3}} \iint d \mathbf{q}_{1} d \mathbf{q}_{2} \delta_{D}\left(\mathbf{q}_{1}+\mathbf{q}_{2}-\mathbf{k}\right) \\
\times \frac{k^{2}\left(\mathbf{q}_{1} \cdot \mathbf{q}_{2}\right)}{2 q_{1}^{2} q_{2}^{2}} \tilde{\theta}_{c}\left(\mathbf{q}_{1}, \tau\right) \tilde{\theta}_{c}\left(\mathbf{q}_{2}, \tau\right),
\end{aligned}
$$

$$
\begin{aligned}
\dot{\tilde{\theta}}_{b}(\mathbf{k}, \tau)+\frac{2}{\tau} \tilde{\theta}_{b}(\mathbf{k}, \tau)+\frac{6}{\tau^{2}} \tilde{\delta}(\mathbf{k}, \tau) \\
=-\frac{1}{(2 \pi)^{3}} \iint d \mathbf{q}_{1} d \mathbf{q}_{2} \delta_{D}\left(\mathbf{q}_{1}+\mathbf{q}_{2}-\mathbf{k}\right) \\
\times \frac{k^{2}\left(\mathbf{q}_{1} \cdot \mathbf{q}_{2}\right)}{2 q_{1}^{2} q_{2}^{2}} \tilde{\theta}_{b}\left(\mathbf{q}_{1}, \tau\right) \tilde{\theta}_{b}\left(\mathbf{q}_{2}, \tau\right) \\
-\mathcal{F}\left[\nabla \cdot\left(\frac{c_{s}^{2}(\tau) \nabla \delta_{b}(\mathbf{x}, \tau)}{1+\delta_{b}(\mathbf{x}, \tau)}\right)\right](\mathbf{k}),
\end{aligned}
$$

where

$$
\begin{aligned}
\mathcal{F}[\nabla & \left.\cdot\left(\frac{c_{s}^{2}(\tau) \nabla \delta_{b}(\mathbf{x}, \tau)}{1+\delta_{b}(\mathbf{x}, \tau)}\right)\right](\mathbf{k}) \\
\equiv & -k^{2} c_{s}^{2}(\tau)\left[\tilde{\delta}_{b}(\mathbf{k})-\frac{1}{2(2 \pi)^{3}} \iint d \mathbf{q}_{1} d \mathbf{q}_{2} \tilde{\delta}_{b}\left(\mathbf{q}_{1}, \tau\right) \tilde{\delta}_{b}\right. \\
& \times\left(\mathbf{q}_{2}, \tau\right) \delta_{D}\left(\mathbf{q}_{1}+\mathbf{q}_{2}-\mathbf{k}\right)+\frac{1}{3(2 \pi)^{6}} \iiint d \mathbf{q}_{1} d \mathbf{q}_{2} d \mathbf{q}_{3} \tilde{\delta}_{b} \\
& \left.\times\left(\mathbf{q}_{1}, \tau\right) \tilde{\delta}_{b}\left(\mathbf{q}_{2}, \tau\right) \tilde{\delta}_{b}\left(\mathbf{q}_{3}, \tau\right) \delta_{D}\left(\mathbf{q}_{1}+\mathbf{q}_{2}+\mathbf{q}_{3}-\mathbf{k}\right)\right] .
\end{aligned}
$$

In the subsequent subsections, we shall solve these coupled equations perturbatively. Hereafter, we shall omit the tildes on the perturbation variables in Fourier space.

\subsection{Linear Order Solution: Jeans Filtering Scale}

In the linear order, one finds

$$
\dot{\delta}_{1, c}(\mathbf{k}, \tau)+\theta_{1, c}(\mathbf{k}, \tau)=0,
$$




$$
\begin{gathered}
\dot{\delta}_{1, b}(\mathbf{k}, \tau)+\theta_{1, b}(\mathbf{k}, \tau)=0, \\
\dot{\theta}_{1, c}(\mathbf{k}, \tau)+\frac{2}{\tau} \theta_{1, c}(\mathbf{k}, \tau)+\frac{6}{\tau^{2}} \delta_{1}(\mathbf{k}, \tau)=0, \\
\dot{\theta}_{1, b}(\mathbf{k}, \tau)+\frac{2}{\tau} \theta_{1, b}(\mathbf{k}, \tau)+\frac{6}{\tau^{2}} \delta_{1}(\mathbf{k}, \tau) \\
-k^{2} c_{s}^{2}(\tau) \delta_{1, b}(\mathbf{k}, \tau)=0,
\end{gathered}
$$

where the subscripts " 1 " mean that these quantities denote the first-order perturbations, and $\delta_{1}=f_{c} \delta_{1, c}+f_{b} \delta_{1, b}$. We rewrite Equation (22) as

$$
\begin{aligned}
& \dot{\theta}_{1, b}(\mathbf{k}, \tau)+\frac{2}{\tau} \theta_{1, b}(\mathbf{k}, \tau)+\frac{6}{\tau^{2}}\left[\delta_{1}(\mathbf{k}, \tau)-\frac{k^{2} c_{s}(\tau)^{2} \tau^{2}}{6} \delta_{1, b}(\mathbf{k}, \tau)\right] \\
& \quad=0, \\
& \dot{\theta}_{1, b}(\mathbf{k}, \tau)+\frac{2}{\tau} \theta_{1, b}(\mathbf{k}, \tau)+\frac{6}{\tau^{2}}\left[\delta_{1}(\mathbf{k}, \tau)-\frac{k^{2}}{k_{J}^{2}} \delta_{1, b}(\mathbf{k}, \tau)\right]=0,
\end{aligned}
$$

where we have used the usual definition of the Jeans wavenumber, $k_{J}$ :

$$
k_{J}(\tau) \equiv \frac{\sqrt{6}}{c_{s}(\tau) \tau} .
$$

The Jeans wavenumber divides the solutions for $\delta_{1, b}$ into two classes: the growing solution for $k \ll k_{J}$, and the oscillatory solution for $k \gg k_{J}$, when there is no $C D M$, i.e., $f_{b}=1$ and $\delta_{1}=\delta_{1, b}$. When $\delta_{1} \neq \delta_{1, b}$, the Jeans wavenumber does not provide a dividing scale for the solutions of $\delta_{1, b}$.

The Jeans wavenumber depends on the temperature of the matter component " $b$ " as $k_{J} \propto T_{b}^{-1 / 2} \tau^{-1}$; thus, $k_{J}$ depends on time in general, $k_{J}=k_{J}(\tau)$. However, in order to simplify the problem and obtain physical insights into the effects of pressure on the nonlinear growth of structure, we shall assume that $k_{J}$ is independent of time, which requires that the matter temperature evolves as if the matter were coupled to radiation, $T_{b} \propto 1 / a \propto 1 / \tau^{2}$. This is not a realistic assumption especially in a low-redshift universe where baryons are decoupled from the radiation background and neutrinos are nonrelativistic-in both cases the temperature evolves as $T_{b} \propto 1 / a^{2} \propto 1 / \tau^{4}$ and thus $k_{J}$ evolves as $k_{J} \propto \tau \propto a^{1 / 2}$, for the adiabatic evolution.

We shall solve the above coupled linear equations iteratively: as CDM is always the most dominant source of gravity, the zeroth-order iterative solution may be found by setting $\delta_{1} \rightarrow \delta_{1, c}$ (i.e., $f_{c} \rightarrow 1$ ). We find the solution for the ratio of the density contrasts, which is often called the "Jeans filtering function" (Gnedin \& Hui 1998)

$$
g_{1}(\mathbf{k}, \tau) \equiv \frac{\delta_{1, b}(\mathbf{k}, \tau)}{\delta_{1, c}(\mathbf{k}, \tau)},
$$

which should be a decreasing function of $k$ due to the effect of pressure. At the zeroth order of iteration, the CDM density contrast grows as

$$
\delta_{1, c}^{(0)}(\mathbf{k}, \tau) \propto a \propto \tau^{2},
$$

and thus the equation for $g_{1}$ simplifies to

$$
\ddot{g}_{1}^{(0)}(\mathbf{k}, \tau)+\frac{6}{\tau} \dot{g}_{1}^{(0)}(\mathbf{k}, \tau)+\frac{6}{\tau^{2}}\left(1+\frac{k^{2}}{k_{J}^{2}}\right) g_{1}^{(0)}(\mathbf{k}, \tau)=\frac{6}{\tau^{2}} .
$$

The solution for $g_{1}(\mathbf{k}, \tau)$ must be normalized such that $g_{1}(k, \tau) \rightarrow 1$ as $k \rightarrow 0$. We find

$$
g_{1}^{(0)}(k, \tau)=\frac{1}{1+\frac{k^{2}}{k_{J}^{2}}}+\mathcal{O}\left(\tau^{m(k)}\right),
$$

where

$$
m(k) \equiv-\frac{5}{2}\left[1 \pm \sqrt{1-\frac{24}{25}\left(1+\frac{k^{2}}{k_{J}^{2}}\right)}\right] .
$$

The second term is a decaying mode, whose amplitude is set by the initial condition, e.g., at the epoch when the baryon temperature was raised (by, say, cosmic re-ionization) to the point where the pressure became important, or at the epoch when the neutrinos became nonrelativistic.

Ignoring the decaying mode (although we shall come back to this later), we have the zeroth-order solution:

$$
g_{1}^{(0)}(k)=\frac{1}{1+\frac{k^{2}}{k_{J}^{2}}} .
$$

At the first-order iteration, we have the pressure feedback on the growth of CDM. The evolution of $\delta_{1, c}^{(1)}$ depends on $k$, and is given by

$$
\delta_{1, c}^{(1)}(k, \tau) \propto \tau^{n(k)}
$$

where

$$
\begin{aligned}
n(k) & \equiv \frac{1}{2}\left[-1 \pm 5 \sqrt{1-\frac{24}{25} f_{b}\left(1-g_{1}^{(0)}(k)\right)}\right] \\
& \simeq\left\{\begin{array}{c}
2-\frac{6}{5} f_{b}\left[1-g_{1}^{(0)}(k)\right] \\
-3+\frac{6}{5} f_{b}\left[1-g_{1}^{(0)}(k)\right]
\end{array} .\right.
\end{aligned}
$$

The second equality is valid for $f_{b}\left[1-g_{1}^{(0)}(k)\right] \ll 1$. The growing mode solution is given by

$$
n_{+}(k)=2-\frac{6}{5} f_{b}\left[1-g_{1}^{(0)}(k)\right] .
$$

As $g^{(0)}(k) \rightarrow 1$ and 0 for $k \rightarrow 0$ and $\infty$, respectively, the largescale and small-scale limits of the growing mode solution is (see, e.g., Section 8.3 of Weinberg 2008 for a recent review)

$$
\begin{aligned}
\delta_{1, c+}^{(1)}(k, \tau) \propto \tau^{2} \propto a, & & k \ll k_{J}, \\
\delta_{1, c+}^{(1)}(k, \tau) \propto \tau^{2-\frac{6}{5} f_{b}} \propto a^{1-\frac{3}{5} f_{b}}, & & k \gg k_{J} .
\end{aligned}
$$

The growth of $\delta_{1, c}$ on the spatial scales below the Jeans scale is suppressed relative to that of the large-scale modes.

Taking the first-order iteration solution for $\delta_{1, c+}^{(1)}$ into account, the first-order iteration equation for $g_{1}^{(1)}$ is

$$
\begin{aligned}
& \ddot{g}_{1}^{(1)}(k, \tau)+\frac{1}{\tau}\left[1+5 \sqrt{1-\frac{24}{25} f_{b}\left(1-g_{1}^{(0)}(k)\right)}\right] \dot{g}_{1}^{(1)}(k, \tau) \\
& +\frac{6}{\tau^{2}}\left[1+\frac{k^{2}}{k_{J}^{2}}-f_{b}\left(2-g_{1}^{(0)}(k)\right)\right] g_{1}^{(1)}(k, \tau)=\frac{6\left(1-f_{b}\right)}{\tau^{2}},
\end{aligned}
$$


whose growing mode solution (with the normalization that $g_{1}^{(1)} \rightarrow 1$ for $\left.k \rightarrow 0\right)$ is

$$
\begin{aligned}
g_{1}^{(1)}(k) & =\frac{1-f_{b}}{1+\frac{k^{2}}{k_{J}^{2}}-f_{b}\left[2-g_{1}^{(0)}(k)\right]} \\
& =\frac{1-f_{b}}{1-f_{b}+\frac{k^{2}}{k_{J}^{2}}\left(1-\frac{f_{b}}{1+k^{2} / k_{J}^{2}}\right)} .
\end{aligned}
$$

This iteration converges quickly for $f_{b}<0.5$, and further iterations are not necessary. The largest difference between $g_{1}^{(0)}(k)$ and $g_{1}^{(1)}(k)$ occurs as $k / k_{J} \rightarrow \infty$, and is $100 \%$ for $f_{b}=0.5$. If the component " $b$ " is identified with baryons, $f_{b} \simeq 1 / 6$, and the difference is reduced to $\sim 20 \%$. The difference between $g_{1}^{(1)}(k)$ and $g_{1}^{(2)}(k)$ occurs at $k \sim k_{J}$, and is $\sim 4 \%$ for $f_{b}=0.5$, and $0.2 \%$ for $f_{b} \simeq 1 / 6$. The difference is much smaller for neutrinos, whose $f_{b}$ are smaller for the modest choices of the neutrino masses $\left(m_{\mathrm{nu}, i}<1 \mathrm{eV}\right)$.

To simplify the subsequent analysis, we shall adopt the zeroth-order iterative solution for the filtering function, $g_{1}^{(0)}=$ $1 /\left(1+k^{2} / k_{J}^{2}\right)$, and the first-order iterative solution for the CDM growth factor, Equation (33), as the solution at the first order in perturbations. This solution is sufficiently accurate for our obtaining the physical insights.

Let us comment on the decaying mode that we have ignored in obtaining Equation (30). This decaying mode is an oscillatory function at $k / k_{J}>1 /(2 \sqrt{6}) \simeq 0.2$, representing the acoustic oscillation of the pressured component (Nusser 2000). While this term is a decaying mode, it decays slowly, and is not quite negligible even at low redshift. We show the decaying mode at the zeroth-order iterative solution in Figure 1,

$$
\Delta g_{1}^{(0)}(k, \tau) \equiv g_{1}^{(0)}(k, \tau)-\frac{1}{1+\frac{k^{2}}{k_{J}^{2}}},
$$

assuming that the pressure became important at $z_{*}=10$. This figure shows that the decaying mode remains important even until $z \sim 0$; thus, technically speaking, ignoring the decaying mode results in an inaccurate form of the filtering function. Nevertheless, we shall ignore it and adopt $g_{1}(k)=1 /\left(1+k^{2} / k_{J}^{2}\right)$.

The exact form of $g_{1}(k, \tau)$ is not so important for our purposes. The main goal of this paper is to study how nonlinearities affect this function. In other words, we are interested in how the higher order filtering functions, $g_{n}(\mathbf{k}, \tau)$, are related to the linear one, $g_{1}(k, \tau)$. One may use any forms of $g_{1}(k, \tau)$ for a better accuracy, depending on the problem (baryons or neutrinos).

\subsection{Second- and Third-order Solutions}

For the higher order ( $n$th order) density perturbations and velocity-divergence fields, we define the Jeans filtering functions such that

$$
\begin{gathered}
g_{n}(\mathbf{k}, \tau) \equiv \frac{\delta_{n, b}(\mathbf{k}, \tau)}{\delta_{n, c}(\mathbf{k}, \tau)}, \\
h_{n}(\mathbf{k}, \tau) \equiv \frac{\theta_{n, b}(\mathbf{k}, \tau)}{\theta_{n, c}(\mathbf{k}, \tau)} .
\end{gathered}
$$

Assuming that CDM dominates the gravitational potential, we find the zeroth-order iteration ansatz in an EdS universe:

$$
\delta_{b}(\mathbf{k}, \tau)=\sum_{n=1}^{\infty} a^{n}(\tau) \delta_{n, c}(\mathbf{k}) g_{n}(\mathbf{k}, \tau)
$$

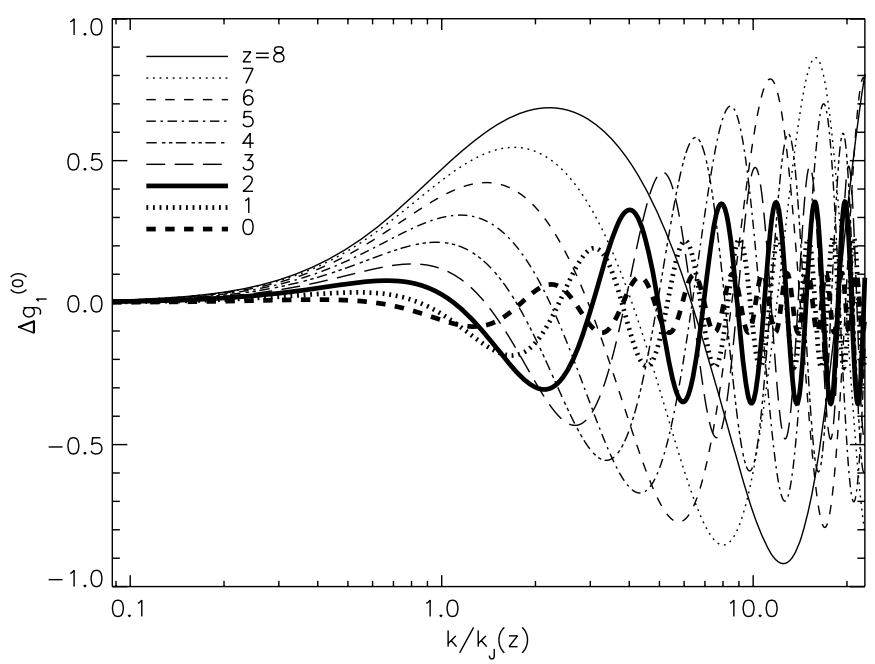

Figure 1. Decaying mode solution for the linear filtering function at the zerothorder iteration $\left(f_{c} \rightarrow 1\right), \Delta g_{1}^{(0)}(k, \tau) \equiv g_{1}^{(0)}(k, \tau)-1 /\left(1+k^{2} / k_{J}^{2}\right)$, where $g_{1}^{(0)}(k, \tau)$ is the numerical solution of Equation (27), with the initial conditions given by $g_{1}^{(0)}\left(k, \tau_{*}\right)=1$ and $\dot{g}_{1}^{(0)}\left(k, \tau_{*}\right)=0$ where $\tau_{*}$ is the conformal time at $z_{*}=10$. The top and bottom lines at $k / k_{J} \sim 1$ are at $z=8$ and 0 , respectively, and the other lines correspond to the intermediate redshifts.

$$
\theta_{b}(\mathbf{k}, \tau)=\sum_{n=1}^{\infty} \dot{a}(\tau) a^{n-1}(\tau) \theta_{n, c}(\mathbf{k}) h_{n}(\mathbf{k}, \tau) .
$$

Detailed derivations of the nonlinear filtering functions at the second order, $g_{2}(\mathbf{k}, \tau)$, and the third order, $g_{3}(\mathbf{k}, \tau)$, are given in Appendix B. The second-order solution is

$$
g_{2}(\mathbf{k}, \tau)=\frac{\frac{10}{3}-\frac{7}{3}\left[1-\frac{\delta_{2, c}^{\prime}(\mathbf{k})}{\delta_{2, c}(\mathbf{k})}\right]}{\frac{10}{3}+\frac{k^{2}}{k_{J}^{2}}}+\mathcal{O}\left(\tau^{-9 / 2}\right),
$$

where

$$
\begin{gathered}
\delta_{2, c}(\mathbf{k})=\frac{1}{(2 \pi)^{3}} \int d \mathbf{q} F_{2}^{(s)}(\mathbf{q}, \mathbf{k}-\mathbf{q}) \delta_{1, c}(\mathbf{q}) \delta_{1, c}(\mathbf{k}-\mathbf{q}), \\
\delta_{2, c}^{\prime}(\mathbf{k})=\frac{1}{(2 \pi)^{3}} \int d \mathbf{q}\left[F_{2}^{(s)}(\mathbf{q}, \mathbf{k}-\mathbf{q})+\frac{3}{14} \frac{k^{2}}{k_{J}^{2}}\right] \\
\times g_{1}(\mathbf{q}) g_{1}(\mathbf{k}-\mathbf{q}) \delta_{1, c}(\mathbf{q}) \delta_{1, c}(\mathbf{k}-\mathbf{q})
\end{gathered}
$$

and $F_{2}^{(s)}$ is a mathematical function given by Equation (A28). The third-order solution is

$$
g_{3}(\mathbf{k}, \tau)=\frac{7-6\left[1-\frac{\delta_{3, c}^{\prime}(\mathbf{k})}{\delta_{3, c}(\mathbf{k})}\right]}{7+\frac{k^{2}}{k_{J}^{2}}}+\mathcal{O}\left(\tau^{-13 / 2}\right),
$$

where

$$
\begin{aligned}
\delta_{3, c}(\mathbf{k})= & \frac{1}{(2 \pi)^{6}} \iiint d \mathbf{q}_{1} d \mathbf{q}_{2} d \mathbf{q}_{3} \delta_{D}\left(\mathbf{k}-\mathbf{q}_{1}-\mathbf{q}_{2}-\mathbf{q}_{3}\right) \\
& \times F_{3}^{(s)}\left(\mathbf{q}_{1}, \mathbf{q}_{2}, \mathbf{q}_{3}\right) \delta_{1, c}\left(\mathbf{q}_{1}\right) \delta_{1, c}\left(\mathbf{q}_{2}\right) \delta_{1, c}\left(\mathbf{q}_{3}\right)
\end{aligned}
$$

$$
\begin{aligned}
\delta_{3, c}^{\prime}(\mathbf{k})= & \frac{1}{(2 \pi)^{6}} \iiint d \mathbf{q}_{1} d \mathbf{q}_{2} d \mathbf{q}_{3} \delta_{D}\left(\mathbf{k}-\mathbf{q}_{1}-\mathbf{q}_{2}-\mathbf{q}_{3}\right) \\
& \times \mathcal{F}_{3}^{(s)}\left(\mathbf{q}_{1}, \mathbf{q}_{2}, \mathbf{q}_{3}\right) \delta_{1, c}\left(\mathbf{q}_{1}\right) \delta_{1, c}\left(\mathbf{q}_{2}\right) \delta_{1, c}\left(\mathbf{q}_{3}\right)
\end{aligned}
$$


and $F_{3}^{(s)}$ and $\mathcal{F}_{3}^{(s)}$ are mathematical functions given by Equations (A30) and (B27), respectively. One may check that these functions are properly normalized, i.e., $g_{n} \rightarrow 1$ as $k \rightarrow 0$, using $\delta_{2, c}^{\prime} \rightarrow \delta_{2, c}$ and $\delta_{3, c}^{\prime} \rightarrow \delta_{3, c}$ as $k \rightarrow 0$.

Ignoring the decaying modes, let us rewrite $g_{2}$ and $g_{3}$ as

$$
\begin{aligned}
& g_{2}(\mathbf{k})=\frac{1-\frac{7}{10}\left[1-\frac{\delta_{2, c}^{\prime}(\mathbf{k})}{\delta_{2, c}(\mathbf{k})}\right]}{1+\frac{3}{10} \frac{k^{2}}{k_{J}^{2}}}, \\
& g_{3}(\mathbf{k})=\frac{1-\frac{6}{7}\left[1-\frac{\delta_{3, c}^{\prime}(\mathbf{k})}{\delta_{3, c}(\mathbf{k})}\right]}{1+\frac{1}{7} \frac{k^{2}}{k_{J}^{2}}} .
\end{aligned}
$$

These results may be interpreted as, roughly speaking, the nonlinear filtering functions having smaller effective filtering scales (larger filtering wavenumbers): $k_{J} \rightarrow \tilde{k}_{J}=\sqrt{\frac{10}{3}} k_{J}$ for the second order, $k_{J} \rightarrow \tilde{k}_{J}=\sqrt{7} k_{J}$ for the third order, and $k_{J} \rightarrow \tilde{k}_{J}=\sqrt{\frac{2}{3} n\left(n+\frac{1}{2}\right)} k_{J}$ for the $n$th order perturbations. In other words, the higher order solutions for $\delta_{n, b}$ are less suppressed relative to the CDM solutions. In the following section, we shall quantify this effect in more detail.

\section{POWER SPECTRUM}

In this section, we calculate the nonlinear matter power spectrum using the results obtained in the previous section. The total matter fluctuation, $\delta$, is given by $\delta=f_{c} \delta_{c}+f_{b} \delta_{b}$, and thus the total matter power spectrum, $P_{\text {tot }}(k)$, is given by the sum of three contributions:

$$
P_{\text {tot }}(k, \tau)=f_{c}^{2} P_{c}(k, \tau)+f_{c} f_{b} P_{b c}(k, \tau)+f_{b}^{2} P_{b}(k, \tau),
$$

where $P_{c}(k)$ and $P_{b}(k)$ are the power spectra of the CDM and another matter component with pressure, respectively, and $P_{b c}(k)$ is the cross-correlation power spectrum. Each term is the sum of the linear part, $P_{11}(k, \tau)$, and the nonlinear parts, $P_{22}(k, \tau)$ and $P_{13}(k, \tau)$ :

$$
P_{i}(k, \tau)=P_{11, i}(k, \tau)+P_{22, i}(k, \tau)+2 P_{13, i}(k, \tau),
$$

where $i=(c, b, b c)$.

The 3PT power spectrum of CDM has been found in the literature (see Bernardeau et al. 2002, for a review)

$$
\begin{aligned}
P_{22, c}(k, \tau)= & 2 \int \frac{d \mathbf{q}}{(2 \pi)^{3}} P_{11, c}(q, \tau) P_{11, c}(|\mathbf{k}-\mathbf{q}|, \tau) \\
& \times\left[F_{2}^{(s)}(\mathbf{q}, \mathbf{k}-\mathbf{q})\right]^{2}
\end{aligned}
$$

where $F_{2}^{(s)}$ is a mathematical function given by Equation (A28), and

$$
\begin{aligned}
P_{13, c}(k, \tau)= & \frac{2 \pi}{252} k^{2} P_{11, c}(k, \tau) \int_{0}^{\infty} \frac{d q}{(2 \pi)^{3}} P_{11, c}(q, \tau) \\
& \times\left[50 \frac{q^{2}}{k^{2}}-21 \frac{q^{4}}{k^{4}}-79+6 \frac{k^{2}}{q^{2}}\right. \\
& \left.+\frac{3}{2} \frac{\left(q^{2}-k^{2}\right)^{3}\left(2 k^{2}+7 q^{2}\right)}{k^{5} q^{3}} \ln \frac{k+q}{|k-q|}\right] .
\end{aligned}
$$

See Appendix A for the detailed derivations.
Here, we have implicitly generalized the results from an EdS universe to general cosmological models, by writing

$$
\begin{aligned}
& \frac{a^{2}(\tau)}{a^{2}\left(\tau_{i}\right)} P_{11}\left(k, \tau_{i}\right) \rightarrow P_{11}(k, \tau) \\
& \quad=\frac{D^{2}(\tau)}{D^{2}\left(\tau_{i}\right)}\left(\frac{\delta_{1, c+}^{(1)}(k, \tau) / \delta_{1, c+}^{(0)}(k, \tau)}{\delta_{1, c+}^{(1)}\left(k, \tau_{*}\right) / \delta_{1, c+}^{(0)}\left(k, \tau_{*}\right)}\right)^{2} P_{11}\left(k, \tau_{i}\right),
\end{aligned}
$$

where $\tau_{i}$ is some arbitrary epoch, $\tau_{*}$ is the epoch where the pressure effect becomes non-negligible (i.e., re-ionization epoch for baryons and the relativistic to nonrelativistic transition epoch for massive neutrinos), and $D(\tau)$ is the linear growth factor appropriate to a given cosmological model. This simple generalization has been shown to provide an excellent approximation to the full calculation: see Bernardeau et al. (2002) for models with nonzero curvature and/or a cosmological constant, and Takahashi (2008) for dynamical dark energy models with a constant equation of state of dark energy.

The linear spectra of the other contributions, $P_{11, b c}$ and $P_{11, b}$, are given by

$$
\begin{gathered}
P_{11, b c}(k, \tau)=g_{1}(k) P_{11, c}(k, \tau), \\
P_{11, b}(k, \tau)=g_{1}^{2}(k) P_{11, c}(k, \tau) .
\end{gathered}
$$

The nonlinear terms, the main results of this paper, are given by

$$
\begin{aligned}
& P_{22, b c}(k, \tau)=\frac{1}{\frac{10}{3}+\frac{k^{2}}{k_{J}^{2}}}\left[P_{22, c}(k, \tau)\right. \\
& +\frac{14}{3} \int \frac{d \mathbf{q}}{(2 \pi)^{3}} P_{11, c}(q, \tau) P_{11, c}(|\mathbf{k}-\mathbf{q}|, \tau) \\
& \left.\times F_{2}^{(s)}(\mathbf{q}, \mathbf{k}-\mathbf{q}) \mathcal{F}_{2}^{(s)}(\mathbf{q}, \mathbf{k}-\mathbf{q})\right] \text {, } \\
& P_{22, b}(k, \tau)=\frac{1}{\left(\frac{10}{3}+\frac{k^{2}}{k_{J}^{2}}\right)^{2}}\left[P_{22, c}(k, \tau)\right. \\
& +\frac{28}{3} \int \frac{d \mathbf{q}}{(2 \pi)^{3}} P_{11, c}(q, \tau) P_{11, c}(|\mathbf{k}-\mathbf{q}|, \tau) \\
& \times F_{2}^{(s)}(\mathbf{q}, \mathbf{k}-\mathbf{q}) \mathcal{F}_{2}^{(s)}(\mathbf{q}, \mathbf{k}-\mathbf{q}) \\
& +\frac{98}{9} \int \frac{d \mathbf{q}}{(2 \pi)^{3}} P_{11, c}(q, \tau) P_{11, c}(|\mathbf{k}-\mathbf{q}|, \tau) \\
& \left.\times\left(\mathcal{F}_{2}^{(s)}(\mathbf{q}, \mathbf{k}-\mathbf{q})\right)^{2}\right] \text {, } \\
& P_{13, b c}(k, \tau)=\frac{1}{2}\left[\left(g_{1}(k)+\frac{1}{7+\frac{k^{2}}{k_{J}^{2}}}\right) P_{13, c}(k, \tau)+\frac{18}{7+\frac{k^{2}}{k_{J}^{2}}} P_{11, c}\right. \\
& \left.\times(k, \tau) \int \frac{d \mathbf{q}}{(2 \pi)^{3}} \mathcal{F}_{3}^{(s)}(\mathbf{q},-\mathbf{q}, \mathbf{k}) P_{11, c}(q, \tau)\right],
\end{aligned}
$$

$$
\begin{aligned}
P_{13, b}(k, \tau)= & \frac{g_{1}(k)}{7+\frac{k^{2}}{k_{J}^{2}}}\left[P_{13, c}(k, \tau)+18 P_{11, c}(k, \tau)\right. \\
& \left.\times \int \frac{d \mathbf{q}}{(2 \pi)^{3}} \mathcal{F}_{3}^{(s)}(\mathbf{q},-\mathbf{q}, \mathbf{k}) P_{11, c}(q, \tau)\right] .
\end{aligned}
$$

See Appendix $\mathrm{C}$ for the detailed derivations. 


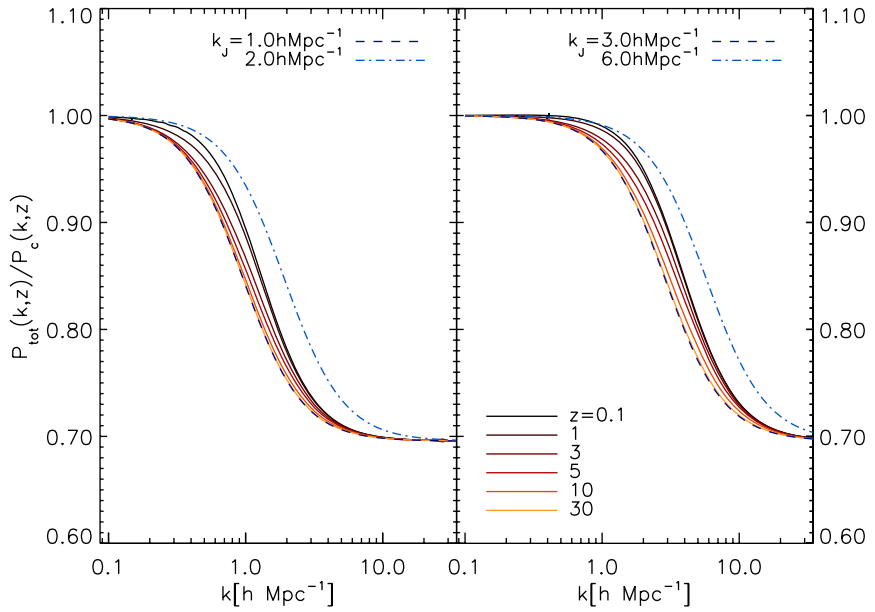

Figure 2. Ratio of the total matter power spectrum, $P_{\text {tot }}(k, z)$, to the CDM part, $P_{c}(k, z)$, at $z=0.1$ (top), 1, 3, 5, 10, and 30 (bottom). Left: the input Jeans wavenumber of $k_{J}=1 h \mathrm{Mpc}^{-1}$. Right: $k_{J}=3 \mathrm{~h} \mathrm{Mpc}^{-1}$. The dashed lines (thin solid lines in the print version) show the ratios calculated from the linear theory, whereas the dot-dashed lines (thin dotted lines in the print version) show the linear calculations with $k_{J}=2$ and $6 \mathrm{~h} \mathrm{Mpc}^{-1}$ for the left and right panels, respectively, to show that the actual filtering wavenumbers, predicted by the 3PT calculations, can be $\sim 40 \%$ as large as the linear filtering wavenumber at low redshift.

(A color version of this figure is available in the online journal.)

How would $P_{\text {tot }}(k)$ compare with the CDM part, $P_{c}(k)$ ?

1. In the linear limit, we should recover $P_{\text {tot }}(k) / P_{c}(k) \rightarrow$ $\left[f_{c}+f_{b} g_{1}(k)\right]^{2}$, which approaches unity as $k \rightarrow 0$.

2 . In the very small scale limit $(k \rightarrow \infty)$, the pressured component is completely smooth $\left(\delta_{b}(k) \rightarrow 0\right)$ because $g_{1}(k) \rightarrow 0$; thus, $P_{\text {tot }}(k) / P_{c}(k)$ approaches a constant value, $f_{c}^{2}$.

3. In the intermediate regime, especially at the transition scale between the super-Jeans scale $\left(k<k_{J}\right)$ and the sub-Jeans scale $\left(k>k_{J}\right)$, the shape of $P_{\text {tot }}(k) / P_{c}(k)$ is significantly distorted away from the linear prediction. Nonlinear clustering of the pressured component adds power at $k \sim k_{J}$, which shifts the effective filtering scale to smaller spatial scales as we go to lower redshifts.

In Figure 2 we show the ratio, $P_{\text {tot }}(k, z) / P_{c}(k, z)$ (solid lines in the online version, thick lines in the print version), for different redshifts $\left(z=0.1,1,3,5,10\right.$, and 30), and different $k_{J}\left(k_{J}=1\right.$ and $3 h \mathrm{Mpc}^{-1}$ for the left and right panels, respectively). In the linear regime (see the bottom lines, $z=30$ ), the ratio agrees with the linear prediction shown by the dashed lines in the online version (thin lines in the print version). As we go to lower redshifts, we find that the filtering wavenumbers continue to shift to larger values, i.e., the filtering scales continue to shift to smaller spatial scales as we go to lower redshifts. This effect cannot be predicted from the linear theory, where all the modes evolve in the same way.

\section{COMPARISON WITH APPROXIMATE TREATMENT OF SAITO ET AL. (2008)}

The nonlinear power spectrum with a significant contribution from a pressured component has not been studied very much in the literature, with one exception. Saito et al. (2008; hereafter STT) have studied effects of massive neutrinos on the nonlinear matter power spectrum using 3PT (also see Wong 2008; Lesgourgues et al. 2009). However, their treatment is not satisfactory: they have entirely ignored nonlinearities in neutrinos, but approximated the neutrino perturbations as linear perturbations. More precisely, they calculated the nonlinear matter power spectrum as

$$
P_{\text {tot }}^{\mathrm{STT}}(k, z)=f_{c}^{2} P_{c}(k, z)+2 f_{c} f_{v} P_{11, v c}(k, z)+f_{v}^{2} P_{11, v}(k, z) .
$$

In our language this leads to

$$
P_{\mathrm{tot}}^{\mathrm{STT}}(k, z)=f_{c}^{2} P_{c}(k)+\left[2 f_{c} f_{\nu} g_{1}(k)+f_{v}^{2} g_{1}^{2}(k)\right] P_{11, c}(k, z) .
$$

Here, we have replaced the subscript " $b$ " with " $v$ " to avoid confusion in notation.

How accurate is the STT approximation? To study this, we compare Equation (63) to the full calculation given in the previous section. Figure 3 shows the fractional difference between our full calculation and STT's approximation, [ $P_{\text {tot }}(k)-$ $\left.P_{\text {tot }}^{\mathrm{STT}}(k)\right] / P_{\text {tot }}(k)$, for $\Omega_{v} / \Omega_{m}=1 / 10,1 / 20$, and $1 / 100$, which correspond to the sum of neutrino masses of $\sum_{i} m_{v, i} \simeq 1.3$, 0.64 , and $0.13 \mathrm{eV}$, respectively, where $i=(e, \mu, \tau)$. We find that STT's approximation clearly underestimates the power at $k \approx k_{\mathrm{FS}}$, where $k_{\mathrm{FS}}$ is the neutrino free-streaming scale, or it is the Jeans wavenumber computed with the velocity dispersion of the neutrinos. More precisely,

$$
k_{\mathrm{FS}, i}(\tau) \equiv \frac{\sqrt{6}}{\sigma_{v, i}(\tau) \tau},
$$

in an EdS universe, where $\sigma_{v, i}^{2}(\tau)$ is the velocity dispersion of neutrino species $i$ (see, e.g., Appendix A.3 of Takada et al. 2006).

One may argue that STT's approximation should be better for a smaller neutrino mass: the errors in the total matter power spectrum are $3.5 \%, 0.6 \%$, and $0.003 \% \sum_{i} m_{v, i}=1.3,0.64$, and $0.13 \mathrm{eV}$, respectively, at $z=0.1$; however, our results indicate that their approximation is conceptually not correct: neutrinos should not be treated as linear perturbations, as the neutrino velocity dispersion has no effect in suppressing the neutrino perturbations at and above the free-streaming scale. In other words, the errors may happen to be small in the total matter power spectrum for small neutrino masses because neutrinos contribute only a tiny fraction of the total matter density anyway, but the errors in the neutrino power spectrum are large. Figure 4 shows the fractional difference between the nonlinear neutrino power spectrum, $P_{v}(k)$, and the linear power spectrum, $P_{v}^{\text {lin }}(k)$, i.e., $\Delta P / P=\left[P_{v}(k)-P_{v}^{\text {lin }}(k)\right] / P_{v}(k)$. It is clear that neutrinos are significantly nonlinear, even well below the free-streaming scale, $k \gg k_{\mathrm{FS}}$. Nevertheless, the STT approximation may still provide a convenient phenomenological tool for calculating the nonlinear total matter power spectrum in the presence of massive neutrinos.

\section{DISCUSSIONS AND CONCLUSIONS}

In this paper, we have obtained the second- and third-order solutions for the density perturbations in a system consisting of two matter components with and without the pressure gradient. This is the first self-consistent analytical calculation, with nonlinearities in the pressured component fully retained up to the third order in perturbations.

As our study is focused on understanding the physics of the nonlinear pressure effect on the matter power spectrum, we have studied a toy model in which the Jeans wavenumber, $k_{J}$, is independent of time. This is equivalent to the temperature of the pressured component following that of radiation, i.e., $T \propto 1 / a$. 


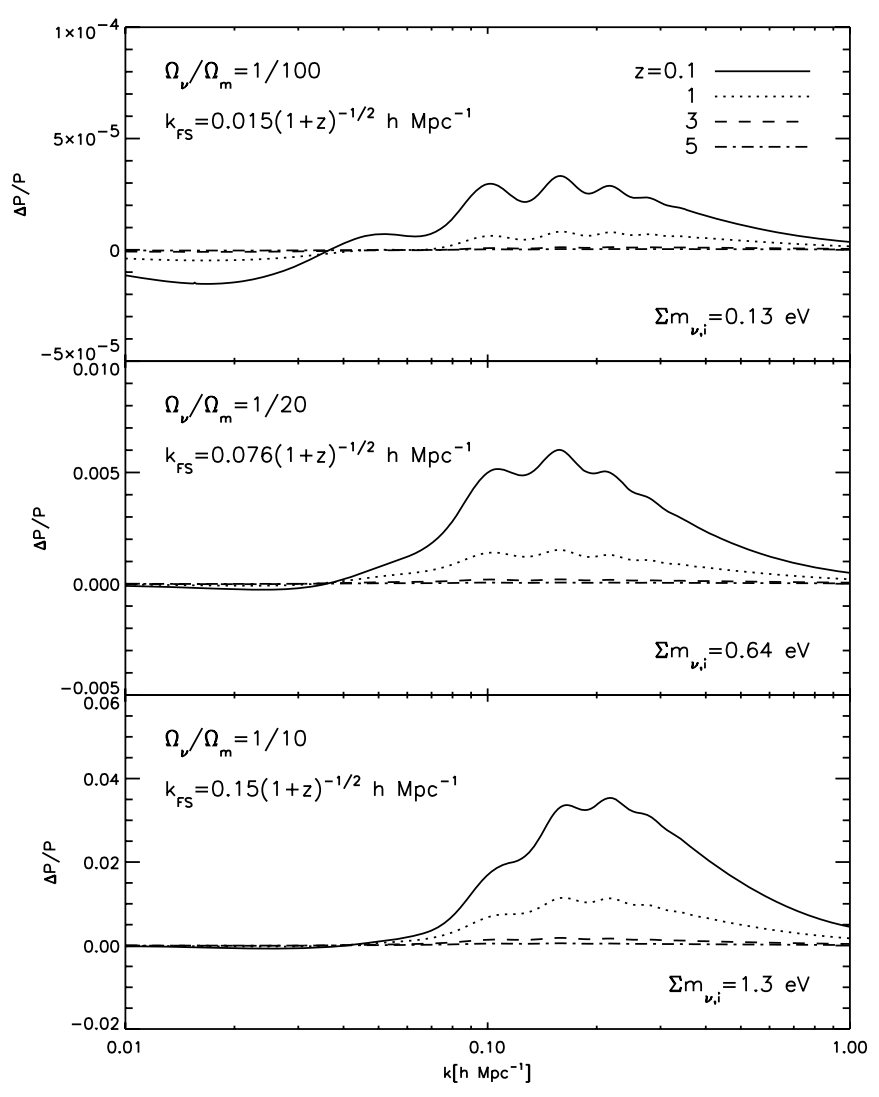

Figure 3. Fractional difference between our full calculation and the approximation used by STT, $\left[P_{\text {tot }}(k)-P_{\text {tot }}^{\mathrm{STT}}(k)\right] / P_{\text {tot }}(k)$, for $\Omega_{v} / \Omega_{m}=1 / 100$ (top), $1 / 20$ (middle), and 1/10 (bottom), which corresponds to $\sum m_{v} \simeq 0.13,0.64$, and $1.3 \mathrm{eV}$, respectively.

Nevertheless, we have found several results that have qualitative implications for the practical applications. We have found that nonlinearities in the pressured component shift the filtering scale from the well known linear filtering scale (Gnedin \& Hui 1998) to a smaller spatial scale (larger wavenumber) by a factor depending on the redshift and the Jeans scale. In other words, the actual filtering scale for a given sound speed (or temperature) is smaller than the linear scale. Therefore, if one used the linear filtering scale to interpret the fall-off of, e.g., the flux power spectrum of the Ly $\alpha$ forests (Zaldarriaga et al. 2001), one would underestimate the temperature of the pressured component.

How important is this effect? For example, when the Jeans

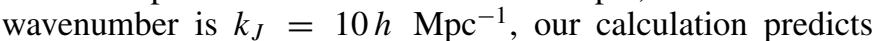
that the effective filtering wavenumber is $\simeq 10,12,13,13$, and $14 \mathrm{~h} \mathrm{Mpc}^{-1}$ at $z=30,10,5,3$, and 1 , respectively. While we do not expect 3PT to be valid at such high wavenumbers, our results clearly indicate that the expected changes in the filtering scale cannot be ignored. Table 1 summarizes the ratios of the effective (actual) and the linear filtering wavenumbers. Note that the linear filtering wavenumber is the same as the Jeans wavenumber in our model; thus, we show $k_{F \text {,eff }} / k_{J}$ in Table 1. We extracted the effective filtering wavenumber, $k_{F, \text { eff }}$, by fitting $\left[f_{c}+f_{b} /\left(1+k^{2} / k_{F, \text { eff }}^{2}\right)\right]^{2}$ to $P_{\text {tot }}(k, z) / P_{c}(k, z)$. We find that a factor of 1.4 change in the filtering scale is quite common over a wide range of redshifts and $k_{J}$.

A factor of 1.4 change in the filtering scale changes the inferred temperature by a factor of 2 ; thus, one implication of our result is that the temperature of the intergalactic medium (IGM) obtained from the Ly $\alpha$ forests at $z=3$ by Zaldarriaga et al. (2001) might have been underestimated by a factor of 2 .

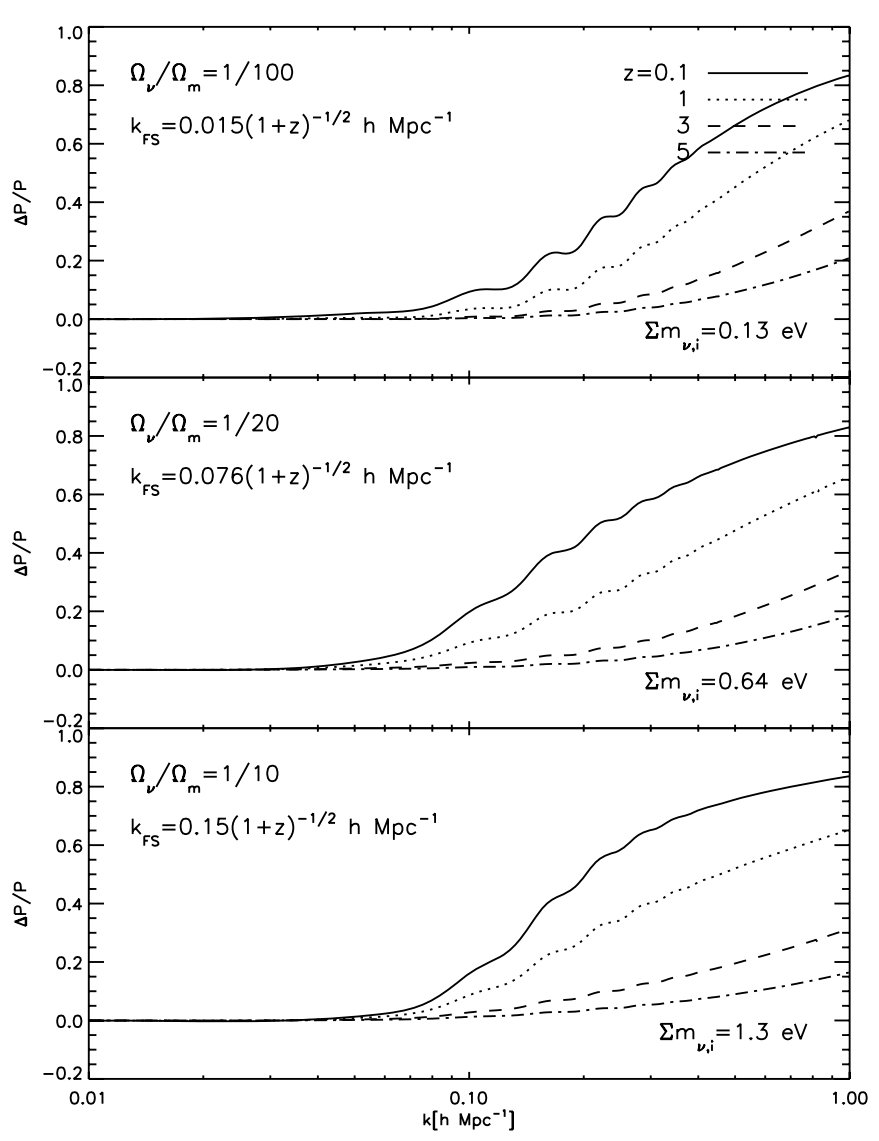

Figure 4. Fractional difference between the nonlinear neutrino power spectrum, $P_{v}(k)$, and the linear power spectrum, $P_{v}^{\operatorname{lin}}(k),\left[P_{v}(k)-P_{v}^{\operatorname{lin}}(k)\right] / P_{v}(k)$, for $\Omega_{v} / \Omega_{m}=1 / 100$ (top), $1 / 20$ (middle), and $1 / 10$ (bottom), which corresponds to $\sum m_{v} \simeq 0.13,0.64$, and $1.3 \mathrm{eV}$, respectively.

Table 1

Ratio of the Effective and the Linear Filtering Scales, $k_{F, \text { eff }} / k_{J}$

\begin{tabular}{lcccccc}
\hline \hline $\begin{array}{l}k_{J} \\
\left(h \mathrm{Mpc}^{-1}\right)\end{array}$ & $z=0.1$ & 1.0 & 3.0 & 5.0 & 10 & 30 \\
\hline 0.1 & 1.08 & 1.04 & 1.01 & 1.00 & 1.00 & 1.00 \\
0.5 & 1.37 & 1.21 & 1.07 & 1.03 & 1.01 & 1.00 \\
1.0 & 1.43 & 1.32 & 1.14 & 1.08 & 1.03 & 1.00 \\
3.0 & 1.41 & 1.38 & 1.28 & 1.20 & 1.08 & 1.01 \\
5.0 & 1.40 & 1.39 & 1.32 & 1.24 & 1.12 & 1.02 \\
10 & 1.41 & 1.40 & 1.35 & 1.29 & 1.16 & 1.03 \\
\hline
\end{tabular}

Notes. This table shows the ratios of the effective $\left(k_{F, \text { eff }}\right)$ and the linear $\left(k_{J}\right)$ filtering scales for different redshifts and $k_{J}$. The ratios are closer to unity at higher redshifts because nonlinearities are weaker.

A factor of 1.4 change in the filtering scale gives a factor of $\sim 3$ change in the filtering mass. Our calculation shows that the actual filtering mass is similar to the linear one only in high redshifts, while the former is significantly smaller than the latter in low redshift. This result is qualitatively similar to those found in Okamoto et al. (2008) and Hoeft et al. (2006); however, a quantitative comparison is not possible, as our results apply only to the system with a constant Jeans wavenumber.

What is next? As for baryons, we need to extend our formalism for incorporating a realistic thermal history of the universe with a proper time dependence of $k_{J}$. As for neutrinos, we need to incorporate not only the pressure gradient but also the anisotropic stress in the Euler equation. To 
do this we need to solve the Boltzmann equation. Nevertheless, our results presented in this paper show that neutrinos are significantly nonlinear, even well below the free-streaming scale.

This material is based in part upon work supported by the Texas Advanced Research Program under Grant 003658-00052006, by NASA grants NNX08AM29G and NNX08AL43G, and by NSF grant AST-0807649. E.K. acknowledges support from an Alfred P. Sloan Research Fellowship.

\section{APPENDIX A}

\section{PT FOR CDM}

The continuity, Euler, and Poisson equations of CDM are given by

1. Continuity equation:

$$
\dot{\delta}(\mathbf{x}, \tau)+\nabla \cdot[(1+\delta(\mathbf{x}, \tau)) \mathbf{v}(\mathbf{x}, \tau)]=0 .
$$

2. Euler equations:

$$
\dot{\mathbf{v}}(\mathbf{x}, \tau)+[\mathbf{v}(\mathbf{x}, \tau) \cdot \nabla] \mathbf{v}(\mathbf{x}, \tau)=-\frac{\dot{a}}{a} \mathbf{v}(\mathbf{x}, \tau)-\nabla \phi(\mathbf{x}, \tau) .
$$

3. Poisson equation (for an EdS universe):

$$
\nabla^{2} \phi(\mathbf{x}, \tau)=\frac{6}{\tau^{2}} \delta(\mathbf{x}, \tau)
$$

First, we take the divergence of Equation (A2) and substitute Equation (A3). Moving all the nonlinear terms to the right-hand side of the equations, we find

$$
\begin{aligned}
& \dot{\delta}(\mathbf{x}, \tau)+\nabla \cdot \mathbf{v}(\mathbf{x}, \tau)=-\nabla \cdot[\delta(\mathbf{x}, \tau) \mathbf{v}(\mathbf{x}, \tau)], \\
& \frac{\partial}{\partial \tau}[\nabla \cdot \mathbf{v}(\mathbf{x}, \tau)]+\frac{\dot{a}}{a}[\nabla \cdot \mathbf{v}(\mathbf{x}, \tau)]+\frac{6}{\tau^{2}} \delta(\mathbf{x}, \tau) \\
& =-\nabla \cdot\{[\mathbf{v}(\mathbf{x}, \tau) \cdot \nabla] \mathbf{v}(\mathbf{x}, \tau)\} .
\end{aligned}
$$

Let us take the Fourier transform of Equations (A4) and (A5)

$$
\begin{gathered}
\dot{\tilde{\delta}}(\mathbf{k}, \tau)+\tilde{\theta}(\mathbf{k}, \tau)=-\frac{1}{(2 \pi)^{3}} \\
\times \iint d \mathbf{q}_{1} d \mathbf{q}_{2} \delta_{D}\left(\mathbf{q}_{1}+\mathbf{q}_{2}-\mathbf{k}\right) \frac{\mathbf{k} \cdot \mathbf{q}_{1}}{q_{1}^{2}} \tilde{\theta}\left(\mathbf{q}_{1}, \tau\right) \tilde{\delta}\left(\mathbf{q}_{2}, \tau\right), \\
\dot{\tilde{\theta}}(\mathbf{k}, \tau)+\frac{\dot{a}}{a} \tilde{\theta}(\mathbf{k}, \tau)+\frac{6}{\tau^{2}} \tilde{\delta}(\mathbf{k}, \tau)=-\frac{1}{(2 \pi)^{3}} \\
\times \iint d \mathbf{q}_{1} d \mathbf{q}_{2} \delta_{D}\left(\mathbf{q}_{1}+\mathbf{q}_{2}-\mathbf{k}\right) \frac{k^{2}\left(\mathbf{q}_{1} \cdot \mathbf{q}_{2}\right)}{2 q_{1}^{2} q_{2}^{2}} \tilde{\theta}\left(\mathbf{q}_{1}, \tau\right) \tilde{\theta}\left(\mathbf{q}_{2}, \tau\right),
\end{gathered}
$$

where we have defined $\theta \equiv \nabla \cdot \mathbf{v}$, and its Fourier transform is given by

$$
\tilde{\mathbf{v}}(\mathbf{k}, \tau)=-i \frac{\mathbf{k}}{k^{2}} \tilde{\theta}(\mathbf{k}, \tau)
$$

One can decompose the solutions of the nonlinear continuity and Euler equations, $\tilde{\delta}$ and $\tilde{\theta}$, into the sum of infinite series of $n$th order perturbations of density and velocity divergence fields:

$$
\tilde{\delta}(\mathbf{k}, \tau)=\sum_{n=1}^{\infty} a^{n}(\tau) \delta_{n}(\mathbf{k}),
$$

$$
\tilde{\theta}(\mathbf{k}, \tau)=\sum_{n=1}^{\infty} \dot{a}(\tau) a^{n-1}(\tau) \theta_{n}(\mathbf{k})
$$

respectively. Note that, strictly speaking, this particular decomposition, a decomposition into a series with powers of $a(\tau)$, is valid only for an EdS universe. However, generalization to arbitrary cosmological models can be done in the end by replacing $a(\tau)$ with the appropriate growth factor, $D(\tau)$ (Bernardeau et al. 2002; Takahashi 2008).

Now, let us solve Equations (A6) and (A7) at each order of perturbations. The $n$th $(n>1)$ term of Equation (A6) is given by

$$
\begin{aligned}
& \dot{a}(\tau) a^{n-1}(\tau)\left[n \delta_{n}(\mathbf{k})+\theta_{n}(\mathbf{k})\right]=-\frac{1}{(2 \pi)^{3}} \iint d \mathbf{q}_{1} d \mathbf{q}_{2} \delta_{D} \\
& \times\left(\mathbf{q}_{1}+\mathbf{q}_{2}-\mathbf{k}\right) \frac{\mathbf{k} \cdot \mathbf{q}_{1}}{q_{1}^{2}} \sum_{m=1}^{n-1} \dot{a}(\tau) a^{n-1}(\tau) \theta_{m}\left(\mathbf{q}_{1}\right) \delta_{n-m}\left(\mathbf{q}_{2}\right) .
\end{aligned}
$$

Dividing both sides by $\dot{a}(\tau) a^{n-1}(\tau)$, one obtains

$$
n \delta_{n}(\mathbf{k})+\theta_{n}(\mathbf{k})=A_{n}(\mathbf{k})
$$

where

$$
\begin{aligned}
A_{n}(\mathbf{k})= & -\frac{1}{(2 \pi)^{3}} \iint d \mathbf{q}_{1} d \mathbf{q}_{2} \delta_{D}\left(\mathbf{q}_{1}+\mathbf{q}_{2}-\mathbf{k}\right) \\
& \times \frac{\mathbf{k} \cdot \mathbf{q}_{1}}{q_{1}^{2}} \sum_{m=1}^{n-1} \theta_{m}\left(\mathbf{q}_{1}\right) \delta_{n-m}\left(\mathbf{q}_{2}\right)
\end{aligned}
$$

Similarly, from the Euler equation, Equation (A7), one obtains

$$
3 \delta_{n}(\mathbf{k})+(1+2 n) \theta_{n}(\mathbf{k})=B_{n}(\mathbf{k}),
$$

where

$$
\begin{aligned}
B_{n}(\mathbf{k})= & -\frac{1}{(2 \pi)^{3}} \iint d \mathbf{q}_{1} d \mathbf{q}_{2} \delta_{D}\left(\mathbf{q}_{1}+\mathbf{q}_{2}-\mathbf{k}\right) \\
& \times \frac{k^{2}\left(\mathbf{q}_{1} \cdot \mathbf{q}_{2}\right)}{q_{1}^{2} q_{2}^{2}} \sum_{m=1}^{n-1} \theta_{m}\left(\mathbf{q}_{1}\right) \theta_{n-m}\left(\mathbf{q}_{2}\right) .
\end{aligned}
$$

The forms of Equations (A12) and (A14) indicate that the $n$th order solutions are written in terms of the sum of first to $(n-1)$ th order solutions, with $\delta_{1}(\mathbf{k})=-\theta_{1}(\mathbf{k})$. By solving Equations (A12) and (A14) for $\delta_{n}$ and $\theta_{n}$, one obtains

$$
\begin{gathered}
\delta_{n}(\mathbf{k})=\frac{(1+2 n) A_{n}(\mathbf{k})-B_{n}(\mathbf{k})}{(2 n+3)(n-1)}, \\
\theta_{n}(\mathbf{k})=\frac{-3 A_{n}(\mathbf{k})+n B_{n}(\mathbf{k})}{(2 n+3)(n-1)},
\end{gathered}
$$

which can be rewritten as

$$
\begin{aligned}
\delta_{n}(\mathbf{k})= & \frac{1}{(2 \pi)^{3 n-3}} \int d \mathbf{q}_{1} \ldots d \mathbf{q}_{n} \delta_{D}\left(\mathbf{q}_{1}+\cdots+\mathbf{q}_{n}-\mathbf{k}\right) \\
& \times F_{n}\left(\mathbf{q}_{1}, \ldots, \mathbf{q}_{n}\right) \delta_{1}\left(\mathbf{q}_{1}\right) \ldots \delta_{1}\left(\mathbf{q}_{n}\right),
\end{aligned}
$$




$$
\begin{aligned}
\theta_{n}(\mathbf{k})= & -\frac{1}{(2 \pi)^{3 n-3}} \int d \mathbf{q}_{1} \ldots d \mathbf{q}_{n} \delta_{D}\left(\mathbf{q}_{1}+\cdots+\mathbf{q}_{n}-\mathbf{k}\right) \\
& \times G_{n}\left(\mathbf{q}_{1}, \ldots, \mathbf{q}_{n}\right) \delta_{1}\left(\mathbf{q}_{1}\right) \ldots \delta_{1}\left(\mathbf{q}_{n}\right) .
\end{aligned}
$$

Here, the newly defined kernels, $F_{n}$ and $G_{n}$, can be found from the following recursion relations:

$$
\begin{aligned}
F_{n}\left(\mathbf{q}_{1}, \ldots, \mathbf{q}_{n}\right)= & \sum_{m=1}^{n-1} \frac{G_{m}\left(\mathbf{q}_{1}, \ldots, \mathbf{q}_{m}\right)}{(2 n+3)(n-1)} \\
& \times\left[(1+2 n) \frac{\mathbf{k} \cdot \mathbf{q}_{1}}{q_{1}^{2}} F_{n-m}\left(\mathbf{q}_{m+1}, \ldots, \mathbf{q}_{n}\right)\right. \\
& \left.\times \frac{k^{2}\left(\mathbf{q}_{1} \cdot \mathbf{q}_{2}\right)}{q_{1}^{2} q_{2}^{2}} G_{n-m}\left(\mathbf{q}_{m+1}, \ldots, \mathbf{q}_{n}\right)\right],
\end{aligned}
$$

and

$$
\begin{aligned}
G_{n}\left(\mathbf{q}_{1}, \ldots, \mathbf{q}_{n}\right)= & \sum_{m=1}^{n-1} \frac{G_{m}\left(\mathbf{q}_{1}, \ldots, \mathbf{q}_{m}\right)}{(2 n+3)(n-1)} \\
& \times\left[3 \frac{\mathbf{k} \cdot \mathbf{q}_{1}}{q_{1}^{2}} F_{n-m}\left(\mathbf{q}_{m+1}, \ldots, \mathbf{q}_{n}\right)\right. \\
& \left.+n \frac{k^{2}\left(\mathbf{q}_{1} \cdot \mathbf{q}_{2}\right)}{q_{1}^{2} q_{2}^{2}} G_{n-m}\left(\mathbf{q}_{m+1}, \ldots, \mathbf{q}_{n}\right)\right],
\end{aligned}
$$

with the boundary conditions of $F_{1}=1=G_{1}$. The secondorder solutions are

$$
\begin{aligned}
F_{2}\left(\mathbf{q}_{1}, \mathbf{q}_{2}\right) & =\frac{5}{7} \frac{\mathbf{k} \cdot \mathbf{q}_{1}}{q_{1}^{2}}+\frac{k^{2}\left(\mathbf{q}_{1} \cdot \mathbf{q}_{2}\right)}{7 q_{1}^{2} q_{2}^{2}}, \\
G_{2}\left(\mathbf{q}_{1}, \mathbf{q}_{2}\right) & =\frac{3}{7} \frac{\mathbf{k} \cdot \mathbf{q}_{1}}{q_{1}^{2}}+\frac{2 k^{2}\left(\mathbf{q}_{1} \cdot \mathbf{q}_{2}\right)}{7 q_{1}^{2} q_{2}^{2}},
\end{aligned}
$$

where $\mathbf{k}=\mathbf{q}_{1}+\mathbf{q}_{2}$. The third-order solutions are

$$
\begin{aligned}
F_{3}\left(\mathbf{q}_{1}, \mathbf{q}_{2}, \mathbf{q}_{3}\right)= & \frac{1}{18}\left[\frac{7 \mathbf{k} \cdot \mathbf{q}_{1}}{q_{1}^{2}} F_{2}\left(\mathbf{q}_{2}, \mathbf{q}_{3}\right)\right. \\
& \left.+\frac{k^{2}\left(\mathbf{q}_{1} \cdot \mathbf{q}_{23}\right)}{q_{1}^{2} q_{23}^{2}} G_{2}\left(\mathbf{q}_{2}, \mathbf{q}_{3}\right)\right]+\frac{G_{2}\left(\mathbf{q}_{1}, \mathbf{q}_{2}\right)}{18} \\
& \times\left[\frac{7 \mathbf{k} \cdot \mathbf{q}_{12}}{q_{12}^{2}}+\frac{k^{2}\left(\mathbf{q}_{12} \cdot \mathbf{q}_{3}\right)}{q_{12}^{2} q_{3}^{2}}\right],
\end{aligned}
$$

where $\mathbf{q}_{i j} \equiv \mathbf{q}_{i}+\mathbf{q}_{j}$ and $\mathbf{k}=\sum \mathbf{q}_{i}$.

It is often convenient to have the symmetrized forms of the above kernels. They are

$$
\begin{gathered}
F_{2}^{(s)}\left(\mathbf{q}_{1}, \mathbf{q}_{2}\right)=\frac{1}{2}\left[F_{2}\left(\mathbf{q}_{1}, \mathbf{q}_{2}\right)+F_{2}\left(\mathbf{q}_{2}, \mathbf{q}_{1}\right)\right], \\
G_{2}^{(s)}\left(\mathbf{q}_{1}, \mathbf{q}_{2}\right)=\frac{1}{2}\left[G_{2}\left(\mathbf{q}_{1}, \mathbf{q}_{2}\right)+G_{2}\left(\mathbf{q}_{2}, \mathbf{q}_{1}\right)\right],
\end{gathered}
$$$$
F_{3}^{(s)}\left(\mathbf{q}_{1}, \mathbf{q}_{2}, \mathbf{q}_{3}\right)=\frac{1}{6}\left[F_{3}\left(\mathbf{q}_{1}, \mathbf{q}_{2}, \mathbf{q}_{3}\right)+F_{3}\left(\mathbf{q}_{1}, \mathbf{q}_{3}, \mathbf{q}_{2}\right)\right.
$$$$
+F_{3}\left(\mathbf{q}_{2}, \mathbf{q}_{1}, \mathbf{q}_{3}\right)+F_{3}\left(\mathbf{q}_{2}, \mathbf{q}_{3}, \mathbf{q}_{1}\right)
$$$$
\left.+F_{3}\left(\mathbf{q}_{3}, \mathbf{q}_{1}, \mathbf{q}_{2}\right)+F_{3}\left(\mathbf{q}_{3}, \mathbf{q}_{2}, \mathbf{q}_{1}\right)\right] \text {. }
$$

The explicit forms are

$$
\begin{aligned}
& F_{2}^{(s)}\left(\mathbf{q}_{1}, \mathbf{q}_{2}\right)=\frac{5}{7}+\frac{2}{7} \frac{\left(\mathbf{q}_{1} \cdot \mathbf{q}_{2}\right)^{2}}{q_{1}^{2} q_{2}^{2}}+\frac{1}{2} \frac{\left(\mathbf{q}_{1} \cdot \mathbf{q}_{2}\right)\left(q_{1}^{2}+q_{2}^{2}\right)}{q_{1}^{2} q_{2}^{2}}, \quad \text { (A28) } \\
& G_{2}^{(s)}\left(\mathbf{q}_{1}, \mathbf{q}_{2}\right)=\frac{3}{7}+\frac{4}{7} \frac{\left(\mathbf{q}_{1} \cdot \mathbf{q}_{2}\right)^{2}}{q_{1}^{2} q_{2}^{2}}+\frac{1}{2} \frac{\left(\mathbf{q}_{1} \cdot \mathbf{q}_{2}\right)\left(q_{1}^{2}+q_{2}^{2}\right)}{q_{1}^{2} q_{2}^{2}}, \quad(\mathrm{~A} 29) \\
& F_{3}^{(s)}\left(\mathbf{q}_{1}, \mathbf{q}_{2}, \mathbf{q}_{3}\right)=\frac{7}{54} \mathbf{k} \cdot\left[F_{2}^{(s)}\left(\mathbf{q}_{2}, \mathbf{q}_{3}\right) \frac{\mathbf{q}_{1}}{q_{1}^{2}}+F_{2}^{(s)}\left(\mathbf{q}_{1}, \mathbf{q}_{3}\right) \frac{\mathbf{q}_{2}}{q_{2}^{2}}\right. \\
& \left.\quad+F_{2}^{(s)}\left(\mathbf{q}_{1}, \mathbf{q}_{2}\right) \frac{\mathbf{q}_{3}}{q_{3}^{2}}\right]+\frac{1}{27} k^{2}\left[G_{2}^{(s)}\left(\mathbf{q}_{2}, \mathbf{q}_{3}\right) \frac{\mathbf{q}_{1} \cdot \mathbf{q}_{23}}{q_{1}^{2} q_{23}^{2}}\right. \\
& \left.\quad+G_{2}^{(s)}\left(\mathbf{q}_{1}, \mathbf{q}_{3}\right) \frac{\mathbf{q}_{2} \cdot \mathbf{q}_{13}}{q_{2}^{2} q_{13}^{2}}+G_{2}^{(s)}\left(\mathbf{q}_{1}, \mathbf{q}_{2}\right) \frac{\mathbf{q}_{3} \cdot \mathbf{q}_{12}}{q_{3}^{2} q_{12}^{2}}\right]+\frac{7}{54} \mathbf{k} \cdot \\
& \quad \times\left[G_{2}^{(s)}\left(\mathbf{q}_{2}, \mathbf{q}_{3}\right) \frac{\mathbf{q}_{23}}{q_{23}^{2}}+G_{2}^{(s)}\left(\mathbf{q}_{1}, \mathbf{q}_{3}\right) \frac{\mathbf{q}_{13}}{q_{13}^{2}}+G_{2}^{(s)}\left(\mathbf{q}_{1}, \mathbf{q}_{2}\right) \frac{\mathbf{q}_{12}}{q_{12}^{2}}\right] .
\end{aligned}
$$

In order to calculate the next-to-linear-order density power spectrum, one needs to use the solutions of the density fluctuations up to the third order:

$$
\begin{array}{rl}
(2 \pi)^{3} & P(k, \tau) \delta_{D}\left(\mathbf{k}+\mathbf{k}^{\prime}\right)=\left\langle\tilde{\delta}(\mathbf{k}, \tau) \tilde{\delta}\left(\mathbf{k}^{\prime}, \tau\right)\right\rangle \\
= & \left\langle\left(\sum_{m=1}^{\infty} a^{m}(\tau) \tilde{\delta}_{m}(\mathbf{k})\right)\left(\sum_{l=1}^{\infty} a^{l}(\tau) \tilde{\delta}_{l}\left(\mathbf{k}^{\prime}\right)\right)\right\rangle \simeq a^{2}(\tau) \\
& \times\left\langle\delta_{1}(\mathbf{k}) \delta_{1}\left(\mathbf{k}^{\prime}\right)\right\rangle+a^{4}(\tau)\left\langle\delta_{1}(\mathbf{k}) \delta_{3}\left(\mathbf{k}^{\prime}\right)\right. \\
& \left.+\delta_{2}(\mathbf{k}) \delta_{2}\left(\mathbf{k}^{\prime}\right)+\delta_{3}(\mathbf{k}) \delta_{1}\left(\mathbf{k}^{\prime}\right)\right\rangle
\end{array}
$$

which yields

$$
P(k, \tau)=a^{2}(\tau) P_{11}(k)+a^{4}(\tau)\left[P_{22}(k)+2 P_{13}(k)\right]+\mathcal{O}\left(\delta^{6}\right) .
$$

Here, we have defined the quantity, $P_{i j}(k)$, given by

$$
(2 \pi)^{3} P_{i j}(k) \delta_{D}\left(\mathbf{k}+\mathbf{k}^{\prime}\right)=\left\langle\delta_{i}(\mathbf{k}) \delta_{j}\left(\mathbf{k}^{\prime}\right)\right\rangle .
$$

The nonlinear corrections, $P_{22}(k)$ and $P_{13}(k)$, are

$$
P_{22}(k)=2 \int \frac{d \mathbf{q}}{(2 \pi)^{3}} P_{11}(q) P_{11}(|\mathbf{k}-\mathbf{q}|)\left[F_{2}^{(s)}(\mathbf{q}, \mathbf{k}-\mathbf{q})\right]^{2},
$$

where

$$
\begin{aligned}
& F_{2}^{(s)}(\mathbf{q}, \mathbf{k}-\mathbf{q})=\frac{5}{7} \\
& \quad+\frac{1}{14}\left[\frac{-10 q^{4}+20 k q^{3} \mu-10 k^{2} q^{2} \mu^{2}-7 k^{2} q^{2}+7 k^{3} q \mu}{q^{2}\left(k^{2}+q^{2}-2 k q \mu\right)}\right]
\end{aligned}
$$

and $\mu \equiv \hat{\mathbf{k}} \cdot \hat{\mathbf{q}}$, and

$$
P_{13}(k)=3 P_{11}(k) \int \frac{d \mathbf{q}}{(2 \pi)^{3}} F_{3}^{(s)}(\mathbf{q},-\mathbf{q}, \mathbf{k}) P_{11}(q) .
$$

Using

$$
\begin{aligned}
\int_{-1}^{1} d \mu F_{3}^{(s)}(\mathbf{q},-\mathbf{q}, \mathbf{k})= & \frac{1}{756}\left[50-21 \frac{q^{2}}{k^{2}}-79 \frac{k^{2}}{q^{2}}+6 \frac{k^{4}}{q^{4}}+\frac{3}{2}\right. \\
& \left.\times \frac{\left(q^{2}-k^{2}\right)^{3}\left(2 k^{2}+7 q^{2}\right)}{k^{3} q^{5}} \ln \frac{k+q}{|k-q|}\right],
\end{aligned}
$$


one obtains (Makino et al. 1992)

$$
\begin{aligned}
P_{13}(k)= & \frac{2 \pi}{252} k^{2} P_{11}(k) \int_{0}^{\infty} \frac{d q}{(2 \pi)^{3}} P_{11}(q)\left[50 \frac{q^{2}}{k^{2}}-21 \frac{q^{4}}{k^{4}}-79\right. \\
& \left.+6 \frac{k^{2}}{q^{2}}+\frac{3}{2} \frac{\left(q^{2}-k^{2}\right)^{3}\left(2 k^{2}+7 q^{2}\right)}{k^{5} q^{3}} \ln \frac{k+q}{|k-q|}\right] . \text { (A37) }
\end{aligned}
$$

\section{APPENDIX B}

\section{PT WITH PRESSURE}

In this appendix, we shall derive the higher order filtering functions. We shall solve Equations (14)-(17) perturbatively, up to the third order in perturbations. The density contrasts and velocity divergence fields of CDM and the matter with pressure are all expanded into the infinite sum of $n$th order perturbations as

$$
\begin{gathered}
\tilde{\delta}_{c}(\mathbf{k}, \tau)=\sum_{n=1}^{\infty} a^{n}(\tau) \delta_{n, c}(\mathbf{k}), \\
\tilde{\theta}_{c}(\mathbf{k}, \tau)=\sum_{n=1}^{\infty} \dot{a}(\tau) a^{n-1}(\tau) \theta_{n, c}(\mathbf{k}), \\
\tilde{\delta}_{b}(\mathbf{k}, \tau)=\sum_{n=1}^{\infty} a^{n}(\tau) \delta_{n, c}(\mathbf{k}) g_{n}(\mathbf{k}, \tau), \\
\tilde{\theta}_{b}(\mathbf{k}, \tau)=\sum_{n=1}^{\infty} \dot{a}(\tau) a^{n-1}(\tau) \theta_{n, c}(\mathbf{k}) h_{n}(\mathbf{k}, \tau),
\end{gathered}
$$

where $g_{n}(\mathbf{k}, \tau)$ and $h_{n}(\mathbf{k}, \tau)$ are the filtering functions for the density and velocity divergence fields, respectively, at the $n$th order.

With the above series expansion, Equations (15) and (17) yield

$$
\begin{aligned}
& \sum_{n=1}^{\infty}[(\left.n \dot{a}(\tau) a^{n-1}(\tau) g_{n}(\mathbf{k}, \tau)+a^{n}(\tau) \dot{g}_{n}(\mathbf{k}, \tau)\right) \\
&\left.\times \delta_{n, c}(\mathbf{k})+\dot{a}(\tau) a^{n-1}(\tau) h_{n}(\mathbf{k}, \tau) \theta_{n, c}(\mathbf{k})\right] \\
&=-\frac{1}{(2 \pi)^{3}} \iint d \mathbf{q}_{1} d \mathbf{q}_{2} \delta_{D}\left(\mathbf{q}_{1}+\mathbf{q}_{2}-\mathbf{k}\right) \frac{\mathbf{k} \cdot \mathbf{q}_{1}}{q_{1}^{2}} \sum_{m=1}^{\infty} \\
& \quad \times \sum_{l=1}^{\infty} \dot{a} a^{m+l-1} h_{m}\left(\mathbf{q}_{1}, \tau\right) g_{l}\left(\mathbf{q}_{2}, \tau\right) \theta_{m, c}\left(\mathbf{q}_{1}\right) \delta_{l, c}\left(\mathbf{q}_{2}\right),
\end{aligned}
$$

$$
\begin{aligned}
& \sum_{n=1}^{\infty}\left[\left(\ddot{a}(\tau) a^{n-1}(\tau)+\dot{a}^{2}(\tau) a^{n-2}(\tau)(n-1)\right) h_{n}(\mathbf{k}, \tau) \theta_{n, c}(\mathbf{k})\right. \\
& +\dot{a}(\tau) a^{n-1}(\tau) \dot{h}_{n}(\mathbf{k}, \tau) \theta_{n, c}(\mathbf{k})+\frac{2}{\tau} \dot{a}(\tau) a^{n-1}(\tau) h_{n}(\mathbf{k})(\mathbf{k}, \tau) \theta_{n, c} \\
& \left.\quad \times(\mathbf{k})+\frac{6}{\tau^{2}} a^{n}(\tau)\left(f_{c}+f_{b} g_{n}(\mathbf{k}, \tau)\right) \delta_{n, c}(\mathbf{k})\right]=-\frac{1}{(2 \pi)^{3}} \\
& \quad \times \iint d \mathbf{q}_{1} d \mathbf{q}_{2} \delta_{D}\left(\mathbf{q}_{1}+\mathbf{q}_{2}-\mathbf{k}\right) \frac{k^{2}\left(\mathbf{q}_{1} \cdot \mathbf{q}_{2}\right)}{2 q_{1}^{2} q_{2}^{2}} \sum_{m=1}^{\infty} \sum_{l=1}^{\infty} \dot{a}^{2}(\tau) \\
& \quad \times a^{m+l-2}(\tau) h_{m}\left(\mathbf{q}_{1}, \tau\right) h_{l}\left(\mathbf{q}_{2}, \tau\right) \theta_{m, c}\left(\mathbf{q}_{1}\right) \theta_{l, c}\left(\mathbf{q}_{2}\right)+k^{2} c_{s}^{2}(\tau)
\end{aligned}
$$

$$
\begin{aligned}
& \times \sum_{n=1}^{\infty} a^{n}(\tau) g_{n}(\mathbf{k}, \tau) \delta_{n, c}(\mathbf{k})-\frac{1}{2(2 \pi)^{3}} k^{2} c_{s}^{2}(\tau) \iint d \mathbf{q}_{1} d \mathbf{q}_{2} \delta_{D} \\
& \times\left(\mathbf{q}_{1}+\mathbf{q}_{2}-\mathbf{k}\right) \sum_{m=1}^{\infty} \sum_{l=1}^{\infty} a^{m+l}(\tau) g_{m}\left(\mathbf{q}_{1}, \tau\right) g_{l}\left(\mathbf{q}_{2}, \tau\right) \delta_{m, c} \\
& \times\left(\mathbf{q}_{1}\right) \delta_{l, c}\left(\mathbf{q}_{2}\right)+\frac{1}{3(2 \pi)^{6}} k^{2} c_{s}^{2}(\tau) \iiint d \mathbf{q}_{1} d \mathbf{q}_{2} d \mathbf{q}_{3} \delta_{D} \\
& \times\left(\mathbf{q}_{1}+\mathbf{q}_{2}+\mathbf{q}_{3}-\mathbf{k}\right) \sum_{m=1}^{\infty} \sum_{l=1}^{\infty} \sum_{p=1}^{\infty} a^{m+l+p}(\tau) g_{m}\left(\mathbf{q}_{1}, \tau\right) g_{l} \\
& \times\left(\mathbf{q}_{2}, \tau\right) g_{p}\left(\mathbf{q}_{3}, \tau\right) \delta_{m, c}\left(\mathbf{q}_{1}\right) \delta_{l, c}\left(\mathbf{q}_{2}\right) \delta_{p, c}\left(\mathbf{q}_{3}\right) .
\end{aligned}
$$

From now on, we shall write the sound speed, $c_{s}$, in terms of the usual Jeans wavenumber, $k_{J}$, as $c_{s}=\sqrt{6} /\left(k_{J} \tau\right)$. We shall ignore the inhomogeneity in $c_{s}$ (i.e., spatial dependence of $c_{s}$ ) throughout this paper. For the linear analysis for $\nabla c_{s} \neq 0$, see Naoz \& Barkana (2005).

\section{B.1. Second-order Solutions}

We have derived the linear filtering function, $g_{1}(k)$, in Equation (30). For $n=2$, the continuity and Euler equations are given by

$$
\begin{aligned}
& \delta_{2, c}(\mathbf{k}) \dot{g}_{2}(\mathbf{k}, \tau)+\frac{4}{\tau} \delta_{2, c}(\mathbf{k}) g_{2}(\mathbf{k}, \tau)+\frac{2}{\tau} \theta_{2, c}(\mathbf{k}) h_{2}(\mathbf{k}, \tau) \\
& =\frac{2}{\tau} \frac{1}{(2 \pi)^{3}} \iint d \mathbf{q}_{1} d \mathbf{q}_{2} \delta_{D}\left(\mathbf{q}_{1}+\mathbf{q}_{2}-\mathbf{k}\right) \\
& \quad \times \frac{\mathbf{k} \cdot \mathbf{q}_{1}}{q_{1}^{2}} \delta_{1, c}\left(\mathbf{q}_{1}\right) \delta_{1, c}\left(\mathbf{q}_{2}\right) g_{1}\left(\mathbf{q}_{1}\right) g_{1}\left(\mathbf{q}_{2}\right) \equiv \frac{2}{\tau} A_{2}(\mathbf{k}),
\end{aligned}
$$

$$
\begin{aligned}
& \frac{10}{\tau^{2}} \theta_{2, c}(\mathbf{k}) h_{2}(\mathbf{k}, \tau)+\frac{2}{\tau} \theta_{2, c}(\mathbf{k}) \dot{h}_{2}(\mathbf{k}, \tau)+\frac{6}{\tau^{2}} \delta_{2, c}(\mathbf{k}) \\
& \quad-\frac{6}{\tau^{2}} \frac{k^{2}}{k_{J}^{2}} \delta_{2, c}(\mathbf{k}) g_{2}(\mathbf{k}, \tau)=\frac{4}{\tau^{2}} \frac{1}{(2 \pi)^{3}} \iint d \mathbf{q}_{1} d \mathbf{q}_{2} \delta_{D} \\
& \quad \times\left(\mathbf{q}_{1}+\mathbf{q}_{2}-\mathbf{k}\right)\left[-\frac{3}{4} \frac{k^{2}}{k_{J}^{2}}-\frac{k^{2}\left(\mathbf{q}_{1} \cdot \mathbf{q}_{2}\right)}{2 q_{1}^{2} q_{2}^{2}}\right] \\
& \quad \times \delta_{1, c}\left(\mathbf{q}_{1}\right) \delta_{1, c}\left(\mathbf{q}_{2}\right) g_{1}\left(\mathbf{q}_{1}\right) g_{1}\left(\mathbf{q}_{2}\right) \equiv \frac{4}{\tau^{2}} B_{2}(\mathbf{k})
\end{aligned}
$$

Here, $\theta_{1, c}(\mathbf{k})=-\delta_{1, c}(\mathbf{k})$. Combining Equations (B7) and (B8), we get the second-order inhomogeneous partial differential equation:

$$
\begin{aligned}
\ddot{g}_{2}(\mathbf{k}, \tau) & +\frac{10}{\tau^{2}} \dot{g}_{2}(\mathbf{k}, \tau)+\frac{1}{\tau^{2}}\left[20+6 \frac{k^{2}}{k_{J}^{2}}\right] g_{2}(\mathbf{k}, \tau) \\
+ & \frac{1}{\tau^{2}}\left[-6-\frac{10 A_{2}(\mathbf{k})}{\delta_{2, c}(\mathbf{k})}+\frac{4 B_{2}(\mathbf{k})}{\delta_{2, c}(\mathbf{k})}\right]=0,
\end{aligned}
$$

where $\delta_{2, c}(\mathbf{k})$ is given by

$$
\delta_{2, c}(\mathbf{k})=\frac{1}{(2 \pi)^{3}} \int d \mathbf{q} F_{2}^{(s)}(\mathbf{q}, \mathbf{k}-\mathbf{q}) \delta_{1, c}(\mathbf{q}) \delta_{1, c}(\mathbf{k}-\mathbf{q}) .
$$

Solving the above differential equation, we have

$$
g_{2}(\mathbf{k}, \tau)=\frac{6+\frac{10 A_{2}(\mathbf{k})}{\delta_{2, c}(\mathbf{k})}-\frac{4 B_{2}(\mathbf{k})}{\delta_{2, c}(\mathbf{k})}}{20+6 \frac{k^{2}}{k_{J}^{2}}}+\mathcal{O}\left(\tau^{-9 / 2}\right),
$$


where the oscillation component,

$$
\mathcal{O}\left(\tau^{-9 / 2}\right) \propto \tau^{-\frac{9}{2}\left(1 \pm \sqrt{1-\frac{4}{81}\left(20+6 \frac{k^{2}}{k_{J}^{2}}\right)}\right)}
$$

decays for any choice of $0 \leqslant k / k_{J}$. The second-order filtering function for the velocity divergence field, $h_{2}(\mathbf{h}, \tau)$, is given by

$$
h_{2}(\mathbf{k})=\frac{1}{\theta_{2, c}(\mathbf{k})}\left[A_{2}(\mathbf{k})-2 \delta_{2, c}(\mathbf{k}) g_{2}(\mathbf{k})\right],
$$

where we have ignored the decaying term.

Using the explicit forms of $A_{2}(\mathbf{k})$ and $B_{2}(\mathbf{k})$ given by Equations (B6) and (B8), respectively, we obtain

$$
g_{2}(\mathbf{k}, \tau)=\frac{\frac{10}{3}-\frac{7}{3}\left[1-\frac{\delta_{2, c}^{\prime}(\mathbf{k})}{\delta_{2, c}(\mathbf{k})}\right]}{\frac{10}{3}+\frac{k^{2}}{k_{J}^{2}}}+\mathcal{O}\left(\tau^{-9 / 2}\right)
$$

where $\delta_{2, c}^{\prime}$ is

$$
\delta_{2, c}^{\prime}(\mathbf{k})=\frac{1}{(2 \pi)^{3}} \int d \mathbf{q} \mathcal{F}_{2}^{(s)}(\mathbf{q}, \mathbf{k}-\mathbf{q}) \delta_{1, c}(\mathbf{q}) \delta_{1, c}(\mathbf{k}-\mathbf{q})
$$

where

$$
\mathcal{F}_{2}^{(s)}\left(\mathbf{q}_{1}, \mathbf{q}_{2}\right) \equiv\left[F_{2}^{(s)}\left(\mathbf{q}_{1}, \mathbf{q}_{2}\right)+\frac{3}{14} \frac{k^{2}}{k_{J}^{2}}\right] g_{1}\left(\mathbf{q}_{1}\right) g_{1}\left(\mathbf{q}_{2}\right) .
$$

In the limit where $k_{J} \rightarrow \infty, \mathcal{F}_{2}^{(s)}\left(\mathbf{q}_{1}, \mathbf{q}_{2}\right)=F_{2}^{(s)}\left(\mathbf{q}_{1}, \mathbf{q}_{2}\right)$, and thus $g_{2} \rightarrow 1$. For the velocity divergence filtering function, we find

$$
\begin{aligned}
h_{2}(\mathbf{k})= & \frac{1}{\theta_{2, c}(\mathbf{k})}\left[\frac{1}{(2 \pi)^{3}} \iint d \mathbf{q}_{1} d \mathbf{q}_{2} \delta_{D}\left(\mathbf{q}_{1}+\mathbf{q}_{2}-\mathbf{k}\right) \delta_{1, c}\left(\mathbf{q}_{1}\right) \delta_{1, c}\right. \\
& \times\left(\mathbf{q}_{2}\right)\left(2 F_{2}^{(s)}\left(\mathbf{q}_{1}, \mathbf{q}_{2}\right)-G_{2}^{(s)}\left(\mathbf{q}_{1}, \mathbf{q}_{2}\right)\right) g_{1}\left(\mathbf{q}_{1}\right) g_{1}\left(\mathbf{q}_{2}\right) \\
& \left.-2 \delta_{2, c}(\mathbf{k}) g_{2}(\mathbf{k})\right]=\frac{1}{\theta_{2, c}(\mathbf{k})}\left[\frac{1}{(2 \pi)^{3}} \iint d \mathbf{q}_{1} d \mathbf{q}_{2} \delta_{D}\right. \\
& \times\left(\mathbf{q}_{1}+\mathbf{q}_{2}-\mathbf{k}\right) \delta_{1, c}\left(\mathbf{q}_{1}\right) \delta_{1, c}\left(\mathbf{q}_{2}\right)\left(1+\frac{\left(\mathbf{q}_{1} \cdot \mathbf{q}_{2}\right)\left(q_{1}^{2}+q_{2}^{2}\right)}{2 q_{1}^{2} q_{2}^{2}}\right) \\
& \left.\times g_{1}\left(\mathbf{q}_{1}\right) g_{1}\left(\mathbf{q}_{2}\right)\right]-2 \frac{\delta_{2, c}(\mathbf{k})}{\theta_{2, c}(\mathbf{k})} g_{2}(\mathbf{k}),
\end{aligned}
$$

where we have used $2 F_{2}\left(\mathbf{q}_{1}, \mathbf{q}_{2}\right)-G_{2}\left(\mathbf{q}_{1}, \mathbf{q}_{2}\right)=\frac{\mathbf{k} \cdot \mathbf{q}_{1}}{q_{1}^{2}}$. This expression also converges to $h_{2}=1$ as we take the limit of $\mathbf{k}_{J} \rightarrow \infty$.

\section{B.2. Third-order Solutions}

For $n=3$, the continuity and Euler equations are given by

$$
\begin{aligned}
& 3 \dot{a}(\tau) a^{2}(\tau) g_{3}(\mathbf{k}, \tau) \delta_{3, c}(\mathbf{k})+a^{3}(\tau) \dot{g}_{3} \\
& \quad \times(\mathbf{k}, \tau) \delta_{3, c}(\mathbf{k})+\dot{a}(\tau) a^{2}(\tau) h_{3}(\mathbf{k}, \tau) \theta_{3, c}(\mathbf{k}) \\
&= \dot{a}(\tau) a^{2}(\tau) \frac{1}{(2 \pi)^{6}} \iiint d \mathbf{q}_{1} d \mathbf{q}_{2} d \mathbf{q}_{3} \delta_{D} \\
& \quad \times\left(\mathbf{q}_{1}+\mathbf{q}_{2}+\mathbf{q}_{3}-\mathbf{k}\right) \delta_{1, c}\left(\mathbf{q}_{1}\right) \delta_{1, c}\left(\mathbf{q}_{2}\right) \delta_{1, c}\left(\mathbf{q}_{3}\right) \\
& \quad \times\left[\frac{\mathbf{k} \cdot \mathbf{q}_{1}}{q_{1}^{2}} g_{1}\left(\mathbf{q}_{1}\right) g_{2}\left(\mathbf{q}_{23}\right) F_{2}^{(s)}\left(\mathbf{q}_{2}, \mathbf{q}_{3}\right)\right. \\
&\left.+\frac{\mathbf{k} \cdot \mathbf{q}_{12}}{q_{12}^{2}} h_{2}\left(\mathbf{q}_{12}\right) g_{1}\left(\mathbf{q}_{3}\right) G_{2}^{(s)}\left(\mathbf{q}_{1}, \mathbf{q}_{2}\right)\right] \equiv \dot{a}(\tau) a^{2}(\tau) A_{3}(\mathbf{k}),
\end{aligned}
$$

$$
\begin{aligned}
& {\left[\ddot{a}(\tau) a^{2}(\tau)+2 \dot{a}^{2}(\tau) a(\tau)\right] h_{3}(\mathbf{k}, \tau) \theta_{3, c}(\mathbf{k})+\dot{a}(\tau) a^{2}(\tau) } \\
& \quad \times \dot{h}_{3}(\mathbf{k}, \tau) \theta_{3, c}(\mathbf{k})+\frac{2}{\tau} \dot{a}(\tau) a^{2}(\tau) h_{3}(\mathbf{k}, \tau) \theta_{3, c}(\mathbf{k}) \\
& \quad+\frac{6}{\tau^{2}} a^{3}(\tau) \delta_{3, c}(\mathbf{k})-\frac{6}{\tau^{2}} \frac{k^{2}}{k_{J}^{2}} a^{3}(\tau) \delta_{3, c}(\mathbf{k}) \\
&=\dot{a}^{2}(\tau) a(\tau) \frac{1}{(2 \pi)^{6}} \iiint d \mathbf{q}_{1} d \mathbf{q}_{2} d \mathbf{q}_{3} \delta_{D} \\
& \quad \times\left(\mathbf{q}_{1}+\mathbf{q}_{2}+\mathbf{q}_{3}-\mathbf{k}\right) \delta_{1, c}\left(\mathbf{q}_{1}\right) \delta_{1, c}\left(\mathbf{q}_{2}\right) \delta_{1, c}\left(\mathbf{q}_{3}\right) \\
& \quad \times\left[-\frac{k^{2}\left(\mathbf{q}_{1} \cdot \mathbf{q}_{23}\right)}{2 q_{1}^{2} q_{23}^{2}} g_{1}\left(\mathbf{q}_{1}\right) h_{2}\left(\mathbf{q}_{23}\right) G_{2}^{(s)}\left(\mathbf{q}_{2}, \mathbf{q}_{3}\right)\right. \\
& \quad-\frac{k^{2}\left(\mathbf{q}_{12} \cdot \mathbf{q}_{3}\right)}{2 q_{12}^{2} q_{3}^{2}} h_{2}\left(\mathbf{q}_{12}\right) g_{1}\left(\mathbf{q}_{3}\right) G_{2}^{(s)}\left(\mathbf{q}_{1}, \mathbf{q}_{2}\right) \\
& \quad-\frac{3}{4} \frac{k^{2}}{k_{J}^{2}} g_{1}\left(\mathbf{q}_{1}\right) g_{2}\left(\mathbf{q}_{23}\right) F_{2}^{(s)}\left(\mathbf{q}_{2}, \mathbf{q}_{3}\right)-\frac{3}{4} \frac{k^{2}}{k_{J}^{2}} g_{2}\left(\mathbf{q}_{12}\right) g_{1}\left(\mathbf{q}_{3}\right) F_{2}^{(s)} \\
&\left.\quad \times\left(\mathbf{q}_{1}, \mathbf{q}_{2}\right)+\frac{1}{2} \frac{k^{2}}{k_{J}^{2}} g_{1}\left(\mathbf{q}_{1}\right) g_{1}\left(\mathbf{q}_{2}\right) g_{1}\left(\mathbf{q}_{3}\right)\right] \\
& \equiv \dot{a}^{2}(\tau) a(\tau) B_{3}(\mathbf{k}) .
\end{aligned}
$$

In an EdS universe, $a(\tau)=\frac{\tau^{2}}{9}$, we have

$$
\begin{aligned}
& \delta_{3, c}(\mathbf{k}) \dot{g}_{3}(\mathbf{k}, \tau)+\frac{6}{\tau} \delta_{3, c}(\mathbf{k}) g_{3}(\mathbf{k}, \tau)+\frac{2}{\tau} \theta_{3, c}(\mathbf{k}) h_{3}(\mathbf{k}, \tau) \\
& =\frac{2}{\tau} A_{3}(\mathbf{k})
\end{aligned}
$$

$$
\begin{gathered}
\frac{14}{\tau^{2}} h_{3}(\mathbf{k}, \tau) \theta_{3, c}(\mathbf{k})+\frac{2}{\tau} \dot{h}_{3}(\mathbf{k}, \tau) \theta_{3, c}(\mathbf{k})+\frac{6}{\tau^{2}} \delta_{3, c}(\mathbf{k}) \\
-\frac{6}{\tau^{2}} \frac{k^{2}}{k_{J}^{2}} \delta_{3, c}(\mathbf{k}) g_{3}(\mathbf{k}, \tau)=\frac{4}{\tau^{2}} B_{3}(\mathbf{k})
\end{gathered}
$$

Combining Equations (B20) and (B21), we have the secondorder differential equation:

$$
\begin{gathered}
\ddot{g}_{3}(\mathbf{k}, \tau)+\frac{14}{\tau} \dot{g}_{3}(\mathbf{k}, \tau)+\frac{1}{\tau^{2}}\left(42+6 \frac{k^{2}}{k_{J}^{2}}\right) g_{3}(\mathbf{k}, \tau) \\
+\frac{1}{\tau^{2}}\left(-6-\frac{14 A_{3}(\mathbf{k})}{\delta_{3, c}(\mathbf{k})}+\frac{4 B_{3}(\mathbf{k})}{\delta_{3, c}(\mathbf{k})}\right)=0 .
\end{gathered}
$$

Solving this, we obtain

$$
g_{3}(\mathbf{k}, \tau)=\frac{1+\frac{7 A_{3}(\mathbf{k})}{3 \delta_{3, c}(\mathbf{k})}-\frac{2 B_{3}(\mathbf{k})}{3 \delta_{3, c}(\mathbf{k})}}{7+\frac{k^{2}}{k_{J}^{2}}}+\mathcal{O}\left(\tau^{-13 / 2}\right),
$$

where the oscillation component,

$$
\mathcal{O}\left(\tau^{-13 / 2}\right) \propto \tau^{-13 / 2\left(1 \pm \sqrt{1-\frac{24}{169}\left(7+\frac{k^{2}}{k_{J}^{2}}\right)}\right)},
$$

decays for any $0 \leqslant \frac{k}{k_{J}}$. The velocity divergence filtering function at the third order is

$$
h_{3}(\mathbf{k})=\frac{1}{\theta_{3, c}(\mathbf{k})}\left[A_{3}(\mathbf{k})-3 \delta_{3, c}(\mathbf{k}) g_{3}(\mathbf{k})\right],
$$

where we have ignored the decaying term. 
Let us rewrite $7 A_{3}(\mathbf{k})-2 B_{3}(\mathbf{k})$ in Equation (B23) as $7 A_{3}(\mathbf{k})-2 B_{3}(\mathbf{k})$

$$
\begin{aligned}
& =\frac{1}{(2 \pi)^{6}} \iiint d \mathbf{q}_{1} d \mathbf{q}_{2} d \mathbf{q}_{3} \delta_{D}\left(\mathbf{q}_{1}+\mathbf{q}_{2}+\mathbf{q}_{3}-\mathbf{k}\right) \delta_{1, c} \\
& \left(\mathbf{q}_{1}\right) \delta_{1, c}\left(\mathbf{q}_{2}\right) \delta_{1, c}\left(\mathbf{q}_{3}\right) \\
& \times\left[\frac{7 \mathbf{k} \cdot \mathbf{q}_{1}}{q_{1}^{2}} g_{1}\left(\mathbf{q}_{1}\right) g_{2}\left(\mathbf{q}_{23}\right) F_{2}^{(s)}\left(\mathbf{q}_{2}, \mathbf{q}_{3}\right)\right. \\
& +\frac{7 \mathbf{k} \cdot \mathbf{q}_{12}}{q_{12}^{2}} h_{2}\left(\mathbf{q}_{12}\right) g_{1}\left(\mathbf{q}_{3}\right) G_{2}^{(s)}\left(\mathbf{q}_{1}, \mathbf{q}_{2}\right) \\
& +\frac{k^{2}\left(\mathbf{q}_{1} \cdot \mathbf{q}_{23}\right)}{q_{1}^{2} q_{23}^{2}} g_{1}\left(\mathbf{q}_{1}\right) h_{2}\left(\mathbf{q}_{23}\right) G_{2}^{(s)}\left(\mathbf{q}_{2}, \mathbf{q}_{3}\right) \\
& +\frac{k^{2}\left(\mathbf{q}_{12} \cdot \mathbf{q}_{3}\right)}{q_{12}^{2} q_{3}^{2}} h_{2}\left(\mathbf{q}_{12}\right) g_{1}\left(\mathbf{q}_{3}\right) G_{2}^{(s)}\left(\mathbf{q}_{1}, \mathbf{q}_{2}\right) \\
& +\frac{3}{2} \frac{k^{2}}{k_{J}^{2}} g_{1}\left(\mathbf{q}_{1}\right) g_{2}\left(\mathbf{q}_{23}\right) F_{2}^{(s)}\left(\mathbf{q}_{2}, \mathbf{q}_{3}\right) \\
& +\frac{3}{2} \frac{k^{2}}{k_{J}^{2}} g_{2}\left(\mathbf{q}_{12}\right) g_{1}\left(\mathbf{q}_{3}\right) F_{2}^{(s)}\left(\mathbf{q}_{1}, \mathbf{q}_{2}\right) \\
& \left.-\frac{k^{2}}{k_{J}^{2}} g_{1}\left(\mathbf{q}_{1}\right) g_{1}\left(\mathbf{q}_{2}\right) g_{1}\left(\mathbf{q}_{3}\right)\right] \\
& \equiv \frac{18}{(2 \pi)^{6}} \iiint d \mathbf{q}_{1} d \mathbf{q}_{2} d \mathbf{q}_{3} \delta_{D}\left(\mathbf{q}_{1}+\mathbf{q}_{2}+\mathbf{q}_{3}-\mathbf{k}\right) \mathcal{F}_{3} \\
& \times\left(\mathbf{q}_{1}, \mathbf{q}_{2}, \mathbf{q}_{3}\right) \delta_{1, c}\left(\mathbf{q}_{1}\right) \delta_{1, c}\left(\mathbf{q}_{2}\right) \delta_{1, c}\left(\mathbf{q}_{3}\right) \\
& \equiv 18 \delta_{3, c}^{\prime}(\mathbf{k}) \text {. }
\end{aligned}
$$

The new kernel, $\mathcal{F}_{3}\left(\mathbf{q}_{1}, \mathbf{q}_{2}, \mathbf{q}_{3}\right)$, can be symmetrized as

$$
\begin{aligned}
& \mathcal{F}_{3}^{(s)}\left(\mathbf{q}_{1}, \mathbf{q}_{2}, \mathbf{q}_{3}\right) \\
&= \frac{1}{6}\left[\mathcal{F}_{3}\left(\mathbf{q}_{1}, \mathbf{q}_{2}, \mathbf{q}_{3}\right)+\mathcal{F}_{3}\left(\mathbf{q}_{1}, \mathbf{q}_{3}, \mathbf{q}_{2}\right)\right. \\
&+\mathcal{F}_{3}\left(\mathbf{q}_{2}, \mathbf{q}_{1}, \mathbf{q}_{3}\right)+\mathcal{F}_{3}\left(\mathbf{q}_{2}, \mathbf{q}_{3}, \mathbf{q}_{1}\right) \\
&\left.+\mathcal{F}_{3}\left(\mathbf{q}_{3}, \mathbf{q}_{1}, \mathbf{q}_{2}\right)+\mathcal{F}_{3}\left(\mathbf{q}_{3}, \mathbf{q}_{2}, \mathbf{q}_{1}\right)\right] \\
&= \frac{7}{54} \mathbf{k} \cdot\left[F_{2}^{(s)}\left(\mathbf{q}_{2}, \mathbf{q}_{3}\right) \frac{\mathbf{q}_{1}}{q_{1}^{2}} g_{1}\left(\mathbf{q}_{1}\right) g_{2}\left(\mathbf{q}_{23}\right)\right. \\
&+F_{2}^{(s)}\left(\mathbf{q}_{1}, \mathbf{q}_{3}\right) \frac{\mathbf{q}_{2}}{q_{2}^{2}} g_{1}\left(\mathbf{q}_{2}\right) g_{2}\left(\mathbf{q}_{13}\right) \\
&\left.+F_{2}^{(s)}\left(\mathbf{q}_{1}, \mathbf{q}_{2}\right) \frac{\mathbf{q}_{3}}{q_{3}^{2}} g_{1}\left(\mathbf{q}_{3}\right) g_{2}\left(\mathbf{q}_{12}\right)\right] \\
&+\frac{1}{27} k^{2}\left[G_{2}^{(s)}\left(\mathbf{q}_{2}, \mathbf{q}_{3}\right) \frac{\mathbf{q}_{1} \cdot \mathbf{q}_{23}}{q_{1}^{2} q_{23}^{2}} g_{1}\left(\mathbf{q}_{1}\right) h_{2}\left(\mathbf{q}_{23}\right)\right. \\
&+G_{2}^{(s)}\left(\mathbf{q}_{1}, \mathbf{q}_{3}\right) \frac{\mathbf{q}_{2} \cdot \mathbf{q}_{13}}{q_{2}^{2} q_{13}^{2}} g_{1}\left(\mathbf{q}_{2}\right) h_{2}\left(\mathbf{q}_{13}\right) \\
&+\frac{1}{18} \frac{k^{2}}{k_{J}^{2}}\left[g_{1}\left(\mathbf{q}_{1}\right) g_{2}\left(\mathbf{q}_{23}\right) F_{2}^{(s)}\left(\mathbf{q}_{2}, \mathbf{q}_{3}\right)\right. \\
&\left.+G_{2}^{(s)}\left(\mathbf{q}_{1}, \mathbf{q}_{2}\right) \frac{\mathbf{q}_{3} \cdot \mathbf{q}_{12}}{q_{3}^{2} q_{12}^{2}} g_{1}\left(\mathbf{q}_{3}\right) h_{2}\left(\mathbf{q}_{12}\right)\right] \\
&+\frac{7}{54} \mathbf{k} \cdot\left[G_{2}^{(s)}\left(\mathbf{q}_{1}, \mathbf{q}_{3}\right)\right. \\
&+G_{2}^{(s)}\left(\mathbf{q}_{2}, \mathbf{q}_{3}\right) \frac{\mathbf{q}_{23}}{q_{23}^{2}} g_{1}\left(\mathbf{q}_{1}\right) h_{2}\left(\mathbf{q}_{23}\right) \\
&\left.\mathbf{q}_{3}\right) \frac{\mathbf{q}_{13}}{q_{13}^{2}} g_{1}\left(\mathbf{q}_{2}\right) h_{2}\left(\mathbf{q}_{13}\right) \\
&\left.\left.\mathbf{q}_{2}\right) \frac{\mathbf{q}_{12}}{q_{12}^{2}} g_{1}\left(\mathbf{q}_{3}\right) h_{2}\left(\mathbf{q}_{12}\right)\right] \\
&\left.+q_{1}\right)
\end{aligned}
$$

$$
\begin{aligned}
& +g_{1}\left(\mathbf{q}_{3}\right) g_{2}\left(\mathbf{q}_{12}\right) F_{2}^{(s)}\left(\mathbf{q}_{1}, \mathbf{q}_{2}\right) \\
& \left.-g_{1}\left(\mathbf{q}_{1}\right) g_{1}\left(\mathbf{q}_{2}\right) g_{1}\left(\mathbf{q}_{3}\right)\right] .
\end{aligned}
$$

In the limit of $k_{J} \rightarrow \infty, \mathcal{F}_{3} \rightarrow F_{3}$, and $g_{3}(k)=1$. Using $\delta_{3, c}^{\prime}(\mathbf{k})$ introduced above, we write $g_{3}$ as

$$
g_{3}(\mathbf{k})=\frac{7-6\left[1-\frac{\delta_{3, c}^{\prime}(\mathbf{k})}{\delta_{3, c}(\mathbf{k})}\right]}{7+\frac{k^{2}}{k_{J}^{2}}} .
$$

\section{APPENDIX C}

\section{PT TOTAL POWER SPECTRUM}

We calculate the power spectrum of the total matter fluctuations, $\delta=f_{c} \delta_{c}+f_{b} \delta_{b}=f_{c} \delta_{c}+\left(1-f_{c}\right) \delta_{b}$, which is given, up to the third order in perturbations, by

$$
\begin{aligned}
\delta(\mathbf{k}, \tau)= & f_{c} \delta_{c}(\mathbf{k}, \tau)+f_{b} \delta_{b}(\mathbf{k}, \tau) \\
= & f_{c}\left[\delta_{1, c}(\mathbf{k}, \tau)+\delta_{2, c}(\mathbf{k}, \tau)+\delta_{3, c}(\mathbf{k}, \tau)\right] \\
& +\left(1-f_{c}\right)\left[\delta_{1, b}(\mathbf{k}, \tau)+\delta_{2, b}(\mathbf{k}, \tau)+\delta_{3, b}(\mathbf{k}, \tau)\right] \\
= & f_{c}\left[\delta_{1, c}(\mathbf{k}, \tau)+\delta_{2, c}(\mathbf{k}, \tau)+\delta_{3, c}(\mathbf{k}, \tau)\right] \\
& +\left(1-f_{c}\right)\left[g_{1}(k) \delta_{1, c}(\mathbf{k}, \tau)+g_{2}(\mathbf{k}) \delta_{2, c}(\mathbf{k}, \tau)\right. \\
& \left.+g_{3}(\mathbf{k}) \delta_{3, c}(\mathbf{k}, \tau)\right] .
\end{aligned}
$$

The power spectrum is

$$
\begin{aligned}
(2 \pi)^{3} P_{\mathrm{tot}}(k) \delta_{D}\left(\mathbf{k}+\mathbf{k}^{\prime}\right)= & \left\langle\delta(\mathbf{k}) \delta\left(\mathbf{k}^{\prime}\right)\right\rangle \\
= & \left\langle\{ f _ { c } \delta _ { c } ( \mathbf { k } ) + ( 1 - f _ { c } ) \delta _ { b } ( \mathbf { k } ) \} \left\{ f_{c} \delta_{c}\left(\mathbf{k}^{\prime}\right)\right.\right. \\
& \left.\left.+\left(1-f_{c}\right) \delta_{b}\left(\mathbf{k}^{\prime}\right)\right\}\right\rangle \\
= & f_{c}^{2}\left\langle\delta_{c}(\mathbf{k}) \delta_{c}\left(\mathbf{k}^{\prime}\right)\right\rangle+2 f_{c}\left(1-f_{c}\right)\left\langle\delta_{b}(\mathbf{k}) \delta_{c}\right. \\
& \left.\left(\mathbf{k}^{\prime}\right)\right\rangle+\left(1-f_{c}\right)^{2}\left\langle\delta_{b}(\mathbf{k}) \delta_{b}\left(\mathbf{k}^{\prime}\right)\right\rangle \\
\equiv & (2 \pi)^{3}\left[f_{c}^{2} P_{c}(k)+2 f_{c}\left(1-f_{c}\right) P_{b, c}(k)\right. \\
& \left.+\left(1-f_{c}\right)^{2} P_{b}(k)\right] \delta_{D}\left(\mathbf{k}+\mathbf{k}^{\prime}\right), \quad(\mathrm{C} 2)
\end{aligned}
$$

where $P_{c}, P_{b, c}$, and $P_{b}$ are

$$
\begin{aligned}
(2 \pi)^{3} P_{c}(k) \delta_{D}\left(\mathbf{k}+\mathbf{k}^{\prime}\right)= & \left\langle\delta_{c}(\mathbf{k}) \delta_{c}\left(\mathbf{k}^{\prime}\right)\right\rangle \\
= & \left\langle\{ \delta _ { 1 , c } ( \mathbf { k } ) + \delta _ { 2 , c } ( \mathbf { k } ) + \delta _ { 3 , c } ( \mathbf { k } ) \} \left\{\delta_{1, c}\left(\mathbf{k}^{\prime}\right)\right.\right. \\
& \left.\left.+\delta_{2, c}\left(\mathbf{k}^{\prime}\right)+\delta_{3, c}\left(\mathbf{k}^{\prime}\right)\right\}\right\rangle \\
= & \left\langle\delta_{1, c}(\mathbf{k}) \delta_{1, c}\left(\mathbf{k}^{\prime}\right)\right\rangle+2\left\langle\delta_{1, c}(\mathbf{k}) \delta_{3, c}\left(\mathbf{k}^{\prime}\right)\right\rangle \\
& +\left\langle\delta_{2, c}(\mathbf{k}) \delta_{2, c}\left(\mathbf{k}^{\prime}\right)\right\rangle \\
\equiv & (2 \pi)^{3}\left[P_{11, c}(k)+2 P_{13, c}(k)+P_{22, c}(k)\right] \\
& \times \delta_{D}\left(\mathbf{k}+\mathbf{k}^{\prime}\right),
\end{aligned}
$$

$$
\begin{aligned}
(2 \pi)^{3} P_{b c}(k) \delta_{D}\left(\mathbf{k}+\mathbf{k}^{\prime}\right)= & \left\langle\delta_{b}(\mathbf{k}) \delta_{c}\left(\mathbf{k}^{\prime}\right)\right\rangle \\
= & \left\langle\left\{ g_{1}(k) \delta_{1, c}(\mathbf{k})+g_{2}(\mathbf{k}) \delta_{2, c}(\mathbf{k})\right.\right. \\
& \left.+g_{3}(\mathbf{k}) \delta_{3, c}(\mathbf{k})\right\}\left\{\delta_{1, c}\left(\mathbf{k}^{\prime}\right)\right. \\
& \left.\left.+\delta_{2, c}\left(\mathbf{k}^{\prime}\right)+\delta_{3, c}\left(\mathbf{k}^{\prime}\right)\right\}\right\rangle \\
= & g_{1}(k)\left\langle\delta_{1, c}(\mathbf{k}) \delta_{1, c}\left(\mathbf{k}^{\prime}\right)\right\rangle \\
& +g_{1}(k)\left\langle\delta_{1, c}(\mathbf{k}) \delta_{3, c}\left(\mathbf{k}^{\prime}\right)\right\rangle \\
& +\left\langle g_{3}(\mathbf{k}) \delta_{3, c}(\mathbf{k}) \delta_{1, c}\left(\mathbf{k}^{\prime}\right)\right\rangle \\
& +\left\langle g_{2}(\mathbf{k}) \delta_{2, c}(\mathbf{k}) \delta_{2, c}\left(\mathbf{k}^{\prime}\right)\right\rangle \\
\equiv & (2 \pi)^{3}\left[P_{11, b c}(k)+2 P_{13, b c}(k)\right. \\
& \left.+P_{22, b c}(k)\right] \delta_{D}\left(\mathbf{k}+\mathbf{k}^{\prime}\right),
\end{aligned}
$$



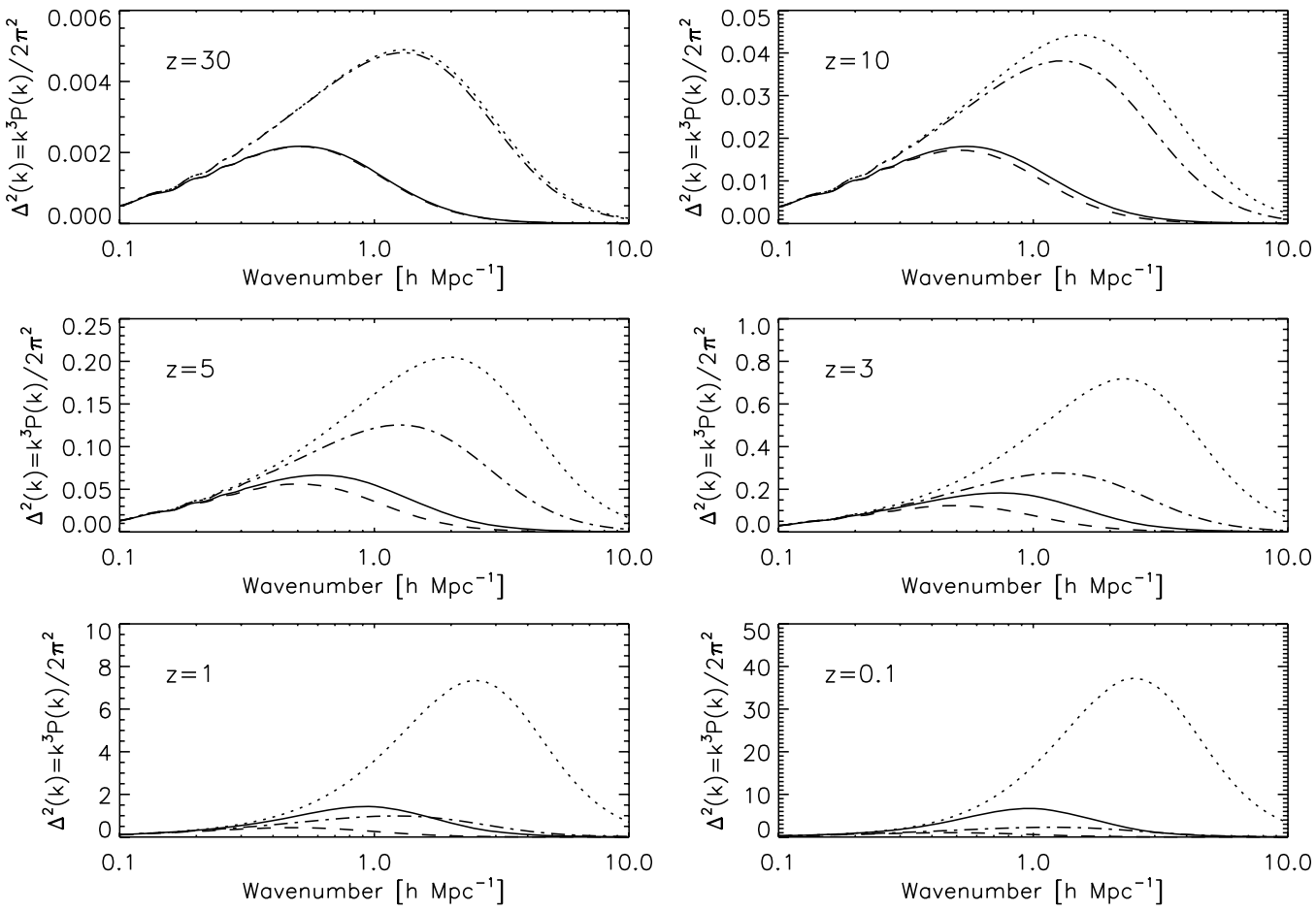

Figure 5. Dimensionless power spectra, $\Delta^{2}(k) \equiv k^{3} P(k) /\left(2 \pi^{2}\right)$, for a matter component with pressure (i.e., baryon, neutrino, etc.) are shown for several redshifts $\left(z=0.1,1.0,3.0,5.0,10\right.$, and 30). We show the nonlinear calculations with 3PT in the solid and dotted lines for $k_{J}=1.0$ and $3.0 h$ Mpc ${ }^{-1}$, respectively. We also show the linear calculations in the dashed and dot-dashed lines for $k_{J}=1.0$ and $3.0 \mathrm{~h} \mathrm{Mpc}{ }^{-1}$, respectively.

$$
\begin{aligned}
(2 \pi)^{3} P_{b}(k) \delta_{D}\left(\mathbf{k}+\mathbf{k}^{\prime}\right)= & \left\langle\delta_{b}(\mathbf{k}) \delta_{b}\left(\mathbf{k}^{\prime}\right)\right\rangle \\
= & \left\langle\left\{ g_{1}(k) \delta_{1, c}(\mathbf{k})+g_{2}(\mathbf{k}) \delta_{2, c}(\mathbf{k})\right.\right. \\
& \left.+g_{3}(\mathbf{k}) \delta_{3, c}(\mathbf{k})\right\} \times\left\{g_{1}\left(k^{\prime}\right) \delta_{1, c}\left(\mathbf{k}^{\prime}\right)\right. \\
& \left.\left.+g_{2}\left(\mathbf{k}^{\prime}\right) \delta_{2, c}\left(\mathbf{k}^{\prime}\right)+g_{3}\left(\mathbf{k}^{\prime}\right) \delta_{3, c}\left(\mathbf{k}^{\prime}\right)\right\}\right\rangle \\
= & g_{1}^{2}(k)\left\langle\delta_{1, c}(\mathbf{k}) \delta_{1, c}\left(\mathbf{k}^{\prime}\right)\right\rangle \\
& +2 g_{1}(k)\left\langle\delta_{1, c}(\mathbf{k}) g_{3}\left(\mathbf{k}^{\prime}\right) \delta_{3, c}\left(\mathbf{k}^{\prime}\right)\right\rangle \\
& +\left\langle g_{2}(\mathbf{k}) \delta_{2, c}(\mathbf{k}) g_{2}\left(\mathbf{k}^{\prime}\right) \delta_{2, c}\left(\mathbf{k}^{\prime}\right)\right\rangle \\
\equiv & (2 \pi)^{3}\left[P_{11, b}(k)+2 P_{13, b}(k)+P_{22, b}(k)\right] \\
& \times \delta_{D}\left(\mathbf{k}+\mathbf{k}^{\prime}\right),
\end{aligned}
$$

respectively.

Now, $P_{11, c}(k), P_{13, c}(k)$, and $P_{22, c}(k)$ can be numerically calculated with the corresponding kernels, $F_{2}^{(s)}$ and $F_{3}^{(s)}$ :

$$
\begin{aligned}
(2 \pi)^{3} P_{11, b c}(k) \delta_{D}\left(\mathbf{k}+\mathbf{k}^{\prime}\right)= & \left\langle\delta_{1, b}(\mathbf{k}) \delta_{1, c}\left(\mathbf{k}^{\prime}\right)\right\rangle \\
& =g_{1}(k)\left\langle\delta_{1, c}(\mathbf{k}) \delta_{1, c}\left(\mathbf{k}^{\prime}\right)\right\rangle, \quad(\mathbf{C} 6) \\
(2 \pi)^{3} P_{13, b c}(k) \delta_{D}\left(\mathbf{k}+\mathbf{k}^{\prime}\right)= & \frac{1}{2}\left[\left\langle\delta_{1, b}(\mathbf{k}) \delta_{3, c}\left(\mathbf{k}^{\prime}\right)\right\rangle\right. \\
& \left.+\left\langle\delta_{1, c}(\mathbf{k}) \delta_{3, b}\left(\mathbf{k}^{\prime}\right)\right\rangle\right] \\
= & \frac{1}{2}\left[g_{1}(k)\left\langle\delta_{1, c}(\mathbf{k}) \delta_{3, c}\left(\mathbf{k}^{\prime}\right)\right\rangle\right. \\
& \left.+\left\langle\delta_{1, c}(\mathbf{k}) g_{3}\left(\mathbf{k}^{\prime}\right) \delta_{3, c}\left(\mathbf{k}^{\prime}\right)\right\rangle\right] \\
= & \frac{1}{2}\left[\left(g_{1}(k)+\frac{1}{\left.7+\frac{k^{2}}{k_{J}^{2}}\right)}\right.\right. \\
& \times\left\langle\delta_{1, c}(\mathbf{k}) \delta_{3, c}\left(\mathbf{k}^{\prime}\right)\right\rangle+\frac{6}{7+\frac{k^{2}}{k_{J}^{2}}} \\
& \left.\times\left\langle\delta_{1, c}(\mathbf{k}) \delta_{3, c}^{\prime}\left(\mathbf{k}^{\prime}\right)\right\rangle\right]
\end{aligned}
$$

$$
\begin{aligned}
(2 \pi)^{3} P_{22, b c}(k) \delta_{D}\left(\mathbf{k}+\mathbf{k}^{\prime}\right)= & \left\langle\delta_{2, b}(\mathbf{k}) \delta_{2, c}\left(\mathbf{k}^{\prime}\right)\right\rangle \\
= & \left\langle g_{2}(\mathbf{k}) \delta_{2, c}(\mathbf{k}) \delta_{2, c}\left(\mathbf{k}^{\prime}\right)\right\rangle \\
= & \frac{1}{\frac{10}{3}+\frac{k^{2}}{k_{J}^{2}}}\left[\left\langle\delta_{2, c}(\mathbf{k}) \delta_{2, c}\left(\mathbf{k}^{\prime}\right)\right\rangle\right. \\
& \left.+\frac{7}{3}\left\langle\delta_{2, c}^{\prime}(\mathbf{k}) \delta_{2, c}\left(\mathbf{k}^{\prime}\right)\right\rangle\right] \\
(2 \pi)^{3} P_{11, b}(k) \delta_{D}\left(\mathbf{k}+\mathbf{k}^{\prime}\right)= & \left\langle\delta_{1, b}(\mathbf{k}) \delta_{1, b}\left(\mathbf{k}^{\prime}\right)\right\rangle \\
= & g_{1}^{2}(k)\left\langle\delta_{1, c}(\mathbf{k}) \delta_{1, c}\left(\mathbf{k}^{\prime}\right)\right\rangle
\end{aligned}
$$

$$
\begin{aligned}
(2 \pi)^{3} P_{13, b}(k) \delta_{D}\left(\mathbf{k}+\mathbf{k}^{\prime}\right)= & \left\langle\delta_{1, b}(\mathbf{k}) \delta_{3, b}\left(\mathbf{k}^{\prime}\right)\right\rangle \\
= & g_{1}(k)\left\langle\delta_{1, c}(\mathbf{k}) g_{3}\left(\mathbf{k}^{\prime}\right) \delta_{3, c}\left(\mathbf{k}^{\prime}\right)\right\rangle \\
= & \frac{g_{1}(k)}{7+\frac{k^{2}}{k_{J}^{2}}}\left[\left\langle\delta_{1, c}(\mathbf{k}) \delta_{3, c}\left(\mathbf{k}^{\prime}\right)\right\rangle\right. \\
& \left.+6\left\langle\delta_{1, c}(\mathbf{k}) \delta_{3, c}^{\prime}\left(\mathbf{k}^{\prime}\right)\right\rangle\right], \\
(2 \pi)^{3} P_{22, b}(k) \delta_{D}\left(\mathbf{k}+\mathbf{k}^{\prime}\right)= & \left\langle\delta_{2, b}(\mathbf{k}) \delta_{2, b}\left(\mathbf{k}^{\prime}\right)\right\rangle \\
= & \left\langle g_{2}(\mathbf{k}) \delta_{2, c}(\mathbf{k}) g_{2}\left(\mathbf{k}^{\prime}\right) \delta_{2, c}\left(\mathbf{k}^{\prime}\right)\right\rangle \\
= & \frac{1}{\left(\frac{10}{3}+\frac{k^{2}}{k_{J}^{2}}\right)^{2}}\left[\left\langle\delta_{2, c}(\mathbf{k}) \delta_{2, c}\left(\mathbf{k}^{\prime}\right)\right\rangle\right. \\
& +\frac{14}{3}\left\langle\delta_{2, c}(\mathbf{k}) \delta_{2, c}^{\prime}\left(\mathbf{k}^{\prime}\right)\right\rangle \\
& \left.+\frac{49}{9}\left\langle\delta_{2, c}^{\prime}(\mathbf{k}) \delta_{2, c}^{\prime}\left(\mathbf{k}^{\prime}\right)\right\rangle\right] .
\end{aligned}
$$


The ensemble averages of the products involving $\delta_{n, c}^{\prime}(\mathbf{k})$ are given by

$$
\begin{aligned}
\left\langle\delta_{1, c}(\mathbf{k}) \delta_{3, c}^{\prime}\left(\mathbf{k}^{\prime}\right)\right\rangle= & 3 \delta_{D}\left(\mathbf{k}+\mathbf{k}^{\prime}\right) P_{11, c}(k) \\
& \times \int d \mathbf{q} \mathcal{F}_{3}^{(s)}(\mathbf{q},-\mathbf{q}, \mathbf{k}) P_{11, c}(q) \\
= & 6 \pi \delta_{D}\left(\mathbf{k}+\mathbf{k}^{\prime}\right) P_{11, c}(k) \int_{0}^{\infty} d q q^{2} P_{11, c}(q) \\
\times & \int_{-1}^{1} d \mu \mathcal{F}_{3}^{(s)}(\mathbf{q},-\mathbf{q}, \mathbf{k}), \\
\left\langle\delta_{2, c}(\mathbf{k}) \delta_{2, c}^{\prime}\left(\mathbf{k}^{\prime}\right)\right\rangle= & 2 \delta_{D}\left(\mathbf{k}+\mathbf{k}^{\prime}\right) \\
& \times \int d \mathbf{q} P_{11, c}(q) P_{11, c}(|\mathbf{k}-\mathbf{q}|) F_{2}^{(s)} \\
& \times(\mathbf{q}, \mathbf{k}-\mathbf{q}) \mathcal{F}_{2}^{(s)}(\mathbf{q}, \mathbf{k}-\mathbf{q}), \\
\left\langle\delta_{2, c}^{\prime}(\mathbf{k}) \delta_{2, c}^{\prime}\left(\mathbf{k}^{\prime}\right)\right\rangle= & 2 \delta_{D}\left(\mathbf{k}+\mathbf{k}^{\prime}\right) \int d \mathbf{q} P_{11, c}(q) P_{11, c}(|\mathbf{k}-\mathbf{q}|) \\
\times & {\left[\mathcal{F}_{2}^{(s)}(\mathbf{q}, \mathbf{k}-\mathbf{q})\right]^{2} . }
\end{aligned}
$$

Here, the term, $\int \frac{d \mathbf{q}}{(2 \pi)^{3}} \mathcal{F}_{3}(\mathbf{q},-\mathbf{q}, \mathbf{k}) P_{11, c}(q)$, in Equation $(\mathrm{C} 12)$ is given by

$$
\begin{aligned}
& \int \frac{d \mathbf{q}}{(2 \pi)^{3}} \mathcal{F}_{3}^{(s)}(\mathbf{q},-\mathbf{q}, \mathbf{k}) P_{11, c}(q) \\
& =\int \frac{d \mathbf{q}}{(2 \pi)^{3}}\left\{\frac { 7 } { 5 4 } \mathbf { k } \cdot \left[F_{2}^{(s)}(-\mathbf{q}, \mathbf{k}) \frac{\mathbf{q}}{q^{2}} g_{1}(\mathbf{q}) g_{2}(\mathbf{k}-\mathbf{q})\right.\right. \\
& \left.-F_{2}^{(s)}(\mathbf{q}, \mathbf{k}) \frac{\mathbf{q}}{q^{2}} g_{1}(\mathbf{q}) g_{2}(\mathbf{k}+\mathbf{q})\right] \\
& +\frac{2}{27} k^{2}\left[F_{2}^{(s)}(-\mathbf{q}, \mathbf{k}) \frac{\mathbf{q} \cdot(\mathbf{k}-\mathbf{q})}{q^{2}(\mathbf{k}-\mathbf{q})^{2}} g_{1}(\mathbf{q}) g_{2}(\mathbf{k}-\mathbf{q})\right. \\
& \left.-F_{2}^{(s)}(\mathbf{q}, \mathbf{k}) \frac{\mathbf{q} \cdot(\mathbf{k}+\mathbf{q})}{q^{2}(\mathbf{k}+\mathbf{q})^{2}} g_{1}(\mathbf{q}) g_{2}(\mathbf{k}+\mathbf{q})\right] \\
& +\frac{14}{54} \mathbf{k} \cdot\left[F_{2}^{(s)}(-\mathbf{q}, \mathbf{k}) \frac{\mathbf{k}-\mathbf{q}}{(\mathbf{k}-\mathbf{q})^{2}} g_{1}(\mathbf{q}) g_{2}(\mathbf{k}-\mathbf{q})\right. \\
& \left.+F_{2}^{(s)}(\mathbf{q}, \mathbf{k}) \frac{\mathbf{k}+\mathbf{q}}{(\mathbf{k}+\mathbf{q})^{2}} g_{1}(\mathbf{q}) g_{2}(\mathbf{k}+\mathbf{q})\right] \\
& -\frac{1}{27} k^{2}\left[\left(1+\frac{(-\mathbf{q} \cdot \mathbf{k})\left(q^{2}+k^{2}\right)}{2 q^{2} k^{2}}\right) \frac{\mathbf{q} \cdot(\mathbf{k}-\mathbf{q})}{q^{2}(\mathbf{k}-\mathbf{q})^{2}} g_{1}^{2}(\mathbf{q}) g_{1}(\mathbf{k})\right. \\
& \left.-\left(1+\frac{(\mathbf{q} \cdot \mathbf{k})\left(q^{2}+k^{2}\right)}{2 q^{2} k^{2}}\right) \frac{\mathbf{q} \cdot(\mathbf{k}+\mathbf{q})}{q^{2}(\mathbf{k}+\mathbf{q})^{2}} g_{1}^{2}(\mathbf{q}) g_{1}(\mathbf{k})\right] \\
& -\frac{7}{54} \mathbf{k} \cdot\left[\left(1+\frac{(-\mathbf{q} \cdot \mathbf{k})\left(q^{2}+k^{2}\right)}{2 q^{2} k^{2}}\right) \frac{\mathbf{k}-\mathbf{q}}{(\mathbf{k}-\mathbf{q})^{2}} g_{1}^{2}(\mathbf{q}) g_{1}(\mathbf{k})\right. \\
& \left.+\left(1+\frac{(\mathbf{q} \cdot \mathbf{k})\left(q^{2}+k^{2}\right)}{2 q^{2} k^{2}}\right) \frac{\mathbf{k}+\mathbf{q}}{(\mathbf{k}+\mathbf{q})^{2}} g_{1}^{2}(\mathbf{q}) g_{1}(\mathbf{k})\right] \\
& +\frac{1}{18} \frac{k^{2}}{k_{J}^{2}}\left[g_{1}(\mathbf{q}) g_{2}(\mathbf{k}-\mathbf{q}) F_{2}^{(s)}(-\mathbf{q}, \mathbf{k})\right. \\
& +g_{1}(\mathbf{q}) g_{2}(\mathbf{k}+\mathbf{q}) F_{2}^{(s)}(\mathbf{q}, \mathbf{k}) \\
& \left.\left.-g_{1}^{2}(\mathbf{q}) g_{1}(\mathbf{k})\right]\right\} P_{11, c}(q)
\end{aligned}
$$

where we have used Equation (B17) and $F_{2}^{(s)}(\mathbf{q},-\mathbf{q})=$ $G_{2}^{(s)}(\mathbf{q},-\mathbf{q})=0$. We then calculate the angular average of $\mathcal{F}_{3}^{(s)}$, i.e., $\int d \mu \mathcal{F}_{3}^{(s)}$, for the linear filtering function of $g_{1}(k)=$ $1 /\left(1+k^{2} / k_{J}^{2}\right)$ :

$$
\begin{aligned}
\int_{-1}^{1} d \mu \mathcal{F}_{3}^{(s)}= & \frac{1}{612360 r^{8} s\left(1+r^{2}\right)\left(r^{2}+s^{2}\right)^{2}}\left[\left[3 0 r ^ { 2 } s ^ { 3 } \left[-14000 s^{6}\right.\right.\right. \\
& +810 r^{10}\left(1+s^{2}\right)+900 r^{2} s^{4}\left(-7+5 s^{2}\right) \\
& +60 r^{4} s^{2}\left(105-125 s^{2}+78 s^{4}\right) \\
& +9 r^{8}\left(321-248 s^{2}+159 s^{4}\right) \\
& \left.+27 r^{6}\left(126-87 s^{2}+70 s^{4}+9 s^{6}\right)\right] \\
& -243 r^{8}\left(-7+5 s^{2}+2 s^{4}\right)\left[5\left(r^{4}+s^{2}\right)\left(-1+s^{2}\right)^{2}\right. \\
& \left.+r^{2}\left(5-5 s^{2}-19 s^{4}+5 s^{6}\right)\right] \ln \frac{1+s}{|1-s|} \\
& +\left[10 s^{2}+3 r^{2}\left(1+s^{2}\right)\right]\left[-35 s^{2}\right. \\
& \left.+3 r^{2}\left(-7+s^{2}\right)\right]\left[-2000 s^{6}+135 r^{8}\left(-1+s^{2}\right)^{2}\right. \\
& +240 r^{4} s^{2}\left(3-4 s^{2}+3 s^{4}\right)+300 r^{2}\left(s^{4}+s^{6}\right) \\
& \left.+27 r^{6}\left(5+5 s^{2}-9 s^{4}+5 s^{6}\right)\right] \\
& \left.\left.\times \frac{1}{2} \ln \left[\frac{10 s^{2}+3 r^{2}(1+s)^{2}}{10 s^{2}+3 r^{2}(1-s)^{2}}\right]\right]\right],
\end{aligned}
$$

where $r \equiv k / k_{J}$ and $s \equiv k / q$. We find that the calculation of $\mathcal{F}_{3}$ is numerically unstable as $k / k_{J} \rightarrow 0(r \rightarrow 0)$. The exact limit of $\mathcal{F}_{3}$ is $\lim _{k / k_{J} \rightarrow 0} \mathcal{F}_{3} \rightarrow F_{3}$, and thus one may replace $\mathcal{F}_{3}$ with $F_{3}$ for a sufficiently small value of $k / k_{J}$.

Finally, we generalize the above results from an EdS universe to general cosmological models, by writing

$$
\begin{aligned}
& \frac{a^{2}(\tau)}{a^{2}\left(\tau_{i}\right)} P_{11}\left(k, \tau_{i}\right) \rightarrow P_{11}(k, \tau)=\frac{D^{2}(\tau)}{D^{2}\left(\tau_{i}\right)} \\
& \quad \times\left(\frac{\delta_{1, c+}^{(1)}(k, \tau) / \delta_{1, c+}^{(0)}(k, \tau)}{\delta_{1, c+}^{(1)}\left(k, \tau_{*}\right) / \delta_{1, c+}^{(0)}\left(k, \tau_{*}\right)}\right)^{2} P_{11}\left(k, \tau_{i}\right),
\end{aligned}
$$

where $\tau_{i}$ is some arbitrary epoch, $\tau_{*}$ is the epoch where the pressure effect becomes non-negligible (i.e., re-ionization epoch for baryons and nonrelativistic transition for massive neutrinos), and $D(\tau)$ is the linear growth factor appropriate to a given cosmological model. We obtain Equation (58) from combining Equations (C8), (C13), and $P_{22, c}$ given by Equation (A34). Similarly, we obtain Equation (59) from combining Equations (C11), (C13), (C14), and $P_{22, c}$, Equation (60) from combining Equations (C7), (C12), and $P_{13, c}$ given by Equation (A36), and Equation (61) from combining Equations (C10), (C12), and $P_{13, c}$.

Figure 5 shows the dimensionless 3PT and linear power spectra, $\Delta^{2}(k)=k^{3} P(k) /\left(2 \pi^{2}\right)$, for a matter component with pressure at different redshifts $(z=0.1,1.0,3.0,5.0,10$, and 30) with $k_{J}=1.0$ and $3.0 \mathrm{~h} \mathrm{Mpc}^{-1}$. The 3PT and linear power spectra are similar at the highest redshift, whereas the 3PT has significantly more power than the linear spectrum at larger wavenumbers as we go to lower redshifts. As a result, the filtering scale for a given linear filtering scale migrates toward larger wavenumbers in lower redshifts.

\section{REFERENCES}

Bernardeau, F., Colombi, S., Gaztañaga, E., \& Scoccimarro, R. 2002, Phys. Rep., 367,1

Gnedin, N. Y. 2000, ApJ, 542, 535 
Gnedin, N. Y., \& Hui, L. 1998, MNRAS, 296, 44

Hoeft, M., Yepes, G., Gottlöber, S., \& Springel, V. 2006, MNRAS, 371, 401

Hu, W., Eisenstein, D. J., \& Tegmark, M. 1998, Phys. Rev. Lett., 80, 5255

Lesgourgues, L., Matarrese, S., Pietroni, M., \& Riotto, A. 2009, arXiv:0901.4550

Makino, N., Sasaki, M., \& Suto, Y. 1992, Phys. Rev. D, 46, 585

Naoz, S., \& Barkana, R. 2005, MNRAS, 362, 1047
Nusser, A. 2000, MNRAS, 317, 902

Okamoto, T., Gao, L., \& Theuns, T. 2008, MNRAS, 390, 920

Saito, S., Takada, M., \& Taruya, A. 2008, Phys. Rev. Lett., 100, 191301

Takada, M., Komatsu, E., \& Futamase, T. 2006, Phys. Rev. D, 73, 083520

Takahashi, R. 2008, Prog. Theor. Phys., 120, 549

Weinberg, S. 2008, Cosmology (Oxford: Oxford Univ. Press)

Wong, Y. Y. Y. 2008, J. Cosmol. Astropart. Phys., JCAP10(2008)035

Zaldarriaga, M., Hui, L., \& Tegmark, M. 2001, ApJ, 557, 519 